\title{
On the Parameterized Complexity of Finding Separators with Non-Hereditary Properties ${ }^{\star}$
}

\author{
Pinar Heggernes ${ }^{1}$, Pim van 't Hof ${ }^{1}$, Dániel Marx², Neeldhara Misra ${ }^{3}$, and Yngve Villanger ${ }^{1}$ \\ ${ }^{1}$ Department of Informatics, University of Bergen, Norway. \\ \{pinar .heggernes|pim.vanthof | yngve.villanger\}@ii.uib.no \\ ${ }^{2}$ Computer and Automation Research Institute, Hungarian Academy of Sciences (MTA SZTAKI), \\ Budapest, Hungary. \\ dmarx@cs.bme.hu \\ 3 The Indian Institute of Science, Bangalore, India. \\ neeldhara@csa.iisc.ernet.in
}

\begin{abstract}
We study the problem of finding small $s-t$ separators that induce graphs having certain properties. It is known that finding a minimum clique $s-t$ separator is polynomial-time solvable (Tarjan 1985), while for example the problems of finding a minimum $s-t$ separator that induces a connected graph or forms an independent set are fixed-parameter tractable when parameterized by the size of the separator (Marx, O'Sullivan and Razgon, ACM Trans. Algor., to appear). Motivated by these results, we study properties that generalize cliques, independent sets, and connected graphs, and determine the complexity of finding separators satisfying these properties. We investigate these problems also on bounded-degree graphs. Our results are as follows:
\end{abstract}

(1) Finding a minimum $c$-connected $s-t$ separator is FPT for $c=2$ and $W[1]$-hard for any $c \geq 3$.

(2) Finding a minimum $s-t$ separator with diameter at most $d$ is $W[1]$-hard for any $d \geq 2$.

(3) Finding a minimum $r$-regular $s-t$ separator is $W[1]$-hard for any $r \geq 1$.

(4) For any decidable graph property, finding a minimum $s-t$ separator with this property is FPT parameterized jointly by the size of the separator and the maximum degree.

(5) Finding a connected $s-t$ separator of minimum size does not have a polynomial kernel, even when restricted to graphs of maximum degree at most 3 , unless NP $\subseteq$ coNP/poly.

In order to prove (1), we show that the natural $c$-connected generalization of the wellknown Steiner Tree problem is FPT for $c=2$ and $W[1]$-hard for any $c \geq 3$.

\section{Introduction}

One of the classic topics in combinatorial optimization and algorithmic graph theory deals with finding cuts and separators in graphs. Recently, the study of this type of problems from a parameterized complexity point of view has attracted a large amount of interest $[5,7,13,17-19$, 23-27]. Given a graph $G$ and two vertices $s$ and $t$ of $G$, a subset of vertices $S \subseteq V(G) \backslash\{s, t\}$ is an $s-t$ separator if $s$ and $t$ appear in different connected components of the graph $G-S$. In separation problems, we are typically looking for small separators $S$. A natural extension of the problem is to demand $G[S]$, i.e., the subgraph induced by $S$, to satisfy a certain property.

* This work is supported by the Research Council of Norway and by the European Research Council (ERC) grant 280152. An extended abstract of this paper was presented at the 38th International Workshop on Graph-Theoretic Concepts in Computer Science (WG 2012) [20]. 
(For convenience, when the graph $G[S]$ has a certain property, we will say that the set $S$ itself also has this property. For example, we say that a set $S \subseteq V(G)$ is 2-connected if $G[S]$ is 2-connected.) A classical result in this direction by Tarjan [28] shows that finding small clique separators is polynomial-time solvable. To our knowledge, this is the only known polynomialtime solvable problem of this type. Therefore, we explore here the problem from the viewpoint of parameterized complexity.

Parameterized complexity associates with every instance of a problem a non-negative integer $k$, called the parameter. Unless specifically stated otherwise, the parameter $k$ in this paper will always be the size of the separator we are looking for. We use $n$ and $m$ to denote the number of vertices and edges, respectively, in the input graph $G$. A parameterized problem is fixedparameter tractable (or FPT) if every instance $(x, k)$ can be solved in time $f(k) \cdot|x|^{O(1)}$ for some function $f$ that only depends on $k[11]$, where $|x|$ is the size of the instance. By showing that a parameterized problem is W[1]-hard, we can give strong evidence that it is unlikely to be FPT; we refer to [11] for more background on parameterized complexity.

For any graph class $\mathcal{G}$, let us consider the following parameterized problem.

$\mathcal{G}$-SEPARATOR
Input: A graph $G$, two vertices $s$ and $t$ of $G$, and an integer $k$.
Parameter: $k$.
Question: Does $G$ have an $s-t$ separator $S$ of size at most $k$ such that
$G[S] \in \mathcal{G} ?$

If $\mathcal{G}$ is the class of complete graphs, then $\mathcal{G}$-SEPARATOR is polynomial-time solvable by the above-mentioned result of Tarjan [28]. Furthermore, Marx et al. [24, 25] showed that the problem is fixed-parameter tractable for many natural classes $\mathcal{G}$. We say that $\mathcal{G}$ is hereditary if, for every graph in $\mathcal{G}$, each of its induced subgraphs also belongs to $\mathcal{G}$.

Theorem 1 ([24, 25]). For any decidable and hereditary graph class $\mathcal{G}$, the $\mathcal{G}$-SEPARATOR problem can be solved in time $f_{\mathcal{G}}(k) \cdot(n+m)$.

For example, by letting $\mathcal{G}$ be the class of all graphs without edges, Theorem 1 shows that finding an independent set of size at most $k$ separating $s$ and $t$ is FPT. The proof is based on a combinatorial statement called Treewidth Reduction Theorem, which shows (roughly speaking) that all the inclusionwise minimal $s-t$ separators lie in a bounded-treewidth part of the graph and hence they can be found efficiently. Note that if $\mathcal{G}$ is hereditary, then we can always assume that the separator is inclusionwise minimal (otherwise we can remove vertices from it without leaving $\mathcal{G}$ ).

Theorem 1 naturally raises the question what the parameterized complexity of the $\mathcal{G}$ SEPARATOR problem is for graph classes $\mathcal{G}$ that are not hereditary. Perhaps the most natural candidate is the class of connected graphs. The Connected SEPARATOR problem of deciding whether a graph $G$ has a connected $s-t$ separator of size at most $k$ has been studied by Marx et al. [25]. Although it is not immediately clear how to apply the Treewidth Reduction Theorem to this problem, Marx et al. [25] managed to extend their framework from [24] to prove the following result.

Theorem 2 ([25]). The CONnECTED SEPARATOR problem can be solved in time $f(k) \cdot(n+m)$.

Our results. Motivated by the results in [24,25], we study the problem of finding small $s-t$ separators satisfying different non-hereditary properties. Let us focus on the three tractable classes mentioned above (connected graphs, complete graphs, edgeless graphs) and try to investigate further related classes. 
As Connected Separator is FPT, it is natural to explore what happens if we require higher-order connectivity. It turns out that finding a $c$-connected $s-t$ separator of size at most $k$ remains FPT also for $c=2$, but becomes $W[1]$-hard for any $c \geq 3$. In order to prove this, we show that the natural $c$-connected generalization of the well-known STEINER TREE problem is FPT for $c=2$ and $W[1]$-hard for any $c \geq 3$. This result could be of independent interest. We find it somewhat surprising that the complexity jump occurs when moving from $c=2$ to $c=3$ (compare this with the results of [9] where the complexity jump occurs when moving from requiring connectivity to requiring 2 -connectivity).

We can generalize the class of complete graphs by considering the class of graphs with diameter at most $d$. We show that the problem of finding an $s-t$ separator of size at most $k$ that induces a graph with diameter at most $d$ in $G$ is $W[1]$-hard for any $d \geq 2$. This is in stark contrast with the case $d=1$, as the problem of finding a clique separator of size at most $k$ is known to be solvable in polynomial time [28].

Edgeless graphs can be thought of as 0-regular graphs. This motivates exploring the problem of finding an $r$-regular $s-t$ separator. We show that, unlike the case where $r=0$, which is FPT by Theorem 1, it is $W[1]$-hard to decide if a graph $G$ has an $r$-regular $s-t$ separator of size at most $k$ for any $r \geq 1$.

All the above results are on general graphs, i.e., graph $G$ can be arbitrary. It comes as no surprise that the problem is much easier restricted to bounded-degree graphs. In particular, finding a small connected separator is FPT due to the fact that a bounded-degree graph contains only a bounded number of small connected sets. More interestingly, we show in Section 4 that for every (not necessarily hereditary) decidable graph class $\mathcal{G}$, the $\mathcal{G}$-SEPARATOR problem can be can be solved in time $h_{\mathcal{G}}(k, \Delta(G)) \cdot m \log n$, where $\Delta(G)$ denotes the maximum degree of $G$. We prove this by showing that the following problem can be solved in time $f(|V(H)|, \Delta(G)) \cdot m \log n$ : Given two graphs $G$ and $H$ and two vertices $s$ and $t$ of $G$, decide whether $G$ has an $s-t$ separator $S$ such that $G[S]$ is isomorphic to $H$. This means that we can solve the $\mathcal{G}$-SEPARATOR problem by simply trying all members $H$ of $\mathcal{G}$ having at most $k$ vertices.

Finally, we investigate the existence of polynomial kernels for the problem of finding small $s-t$ separators. A parameterized problem is said to admit a kernel if there is a polynomialtime algorithm that transforms each instance of the problem into an equivalent instance whose size and parameter value are bounded from above by $g(k)$ for some (possibly exponential) function $g$. It is known that a parameterized problem is FPT if and only if it is decidable and admits a kernel [11]. In the desirable case that $g(k)$ is a polynomial in $k$, we say that the problem admits a polynomial kernel. Many problems have been shown to admit polynomial kernels, including classes of problems that are covered by some kernelization meta-theorems [3, 15]. Recently developed methods for proving non-existence of polynomial kernels, up to some complexity theoretical assumptions $[2,4,16]$, significantly contributed to the establishment of kernelization as an important and rapidly growing subfield of parameterized complexity.

Although the CONNECTED SEPARATOR problem is FPT by Theorem 2 and therefore admits a kernel [11], we show in Section 5 that this problem does not admit a polynomial kernel, even when restricted to input graphs of maximum degree at most 3, unless NP $\subseteq$ coNP/poly. This means that techniques other than kernelization (e.g., treewidth reduction) seem to be essential for the efficient solution of the problem even on bounded-degree graphs.

\section{Finding $s-t$ Separators with Higher Connectivity}

Theorem 2 states that the problem of finding a connected $s-t$ separator of size at most $k$ is FPT. In this section, we study the parameterized complexity of finding $s-t$ separators of 
higher connectivity. A graph $G$ is c-connected if $|V(G)|>c$ and $G-X$ is connected for every $X \subseteq V(G)$ with $|X|<c$. For any integer $c \geq 1$, the $c$-Connected Separator problem takes as input a graph $G$, two vertices $s$ and $t$ of $G$, and an integer $k$ (the parameter), and asks whether there is an $s-t$ separator of size at most $k$ that induces a $c$-connected graph. Theorem 2 states that this problem is FPT when $c=1$. Interestingly, it turns out that the problem remains FPT for $c=2$, but becomes $W[1]$-hard for any $c \geq 3$.

The algorithm in [25] for finding a minimum connected $s-t$ separator uses an FPT algorithm for Steiner Tree as a subroutine. Let us recall the definition of the Steiner Tree problem.

STEINER TREE
Input: A graph $G$, a set $T \subseteq V(G)$ of terminals, and an integer $k$.
Parameter: $k$.
Question: Does $G$ have a connected subgraph $H$ on at most $k$ vertices such
that $T \subseteq V(H)$ ?

The Steiner Tree problem is well-known to be NP-complete [22]. Dreyfuss and Wagner [12] showed that the problem is FPT; in fact, they showed that the problem is FPT even when $|T|$ is chosen as the parameter instead of $k$. The following problem, defined for any integer $c \geq 1$, is a natural generalization of the STEINER TREE problem:

$c$-CONNECTED STEINER

Input: A graph $G$, a set $T \subseteq V(G)$ of terminals, and an integer $k$.

Parameter: $k$.

Question: Does $G$ have a $c$-connected subgraph $H$ on at most $k$ vertices such that $T \subseteq V(H)$ ?

A solution $H$ to an instance $(G, T, k)$ of the $c$-Connected Steiner problem is minimal if no proper subgraph of $H$ is a solution, and $H$ is minimum if there is no solution $H^{\prime}$ with $\left|V\left(H^{\prime}\right)\right|<|V(H)|$. The $c$-ConneCTED SteIneR problem is FPT when $c=1$, as the problem is then equivalent to Steiner Tree. We show below that the $c$-Connected Steiner problem remains FPT when $c=2$, but becomes $W[1]$-hard for higher values of $c$.

Let us first describe two very simple polynomial-time reductions that imply NP-completeness of the problems studied in this section. We will also use these two reductions in Section 5 to show that none of these problems admits a polynomial kernel. For any $c \geq 1$, the $c$-ConNECTED STEINER problem is NP-complete, as any instance of STEINER TREE can be transformed into an equivalent instance of $c$-CONNECTED STEINER by adding $c-1$ universal vertices to the terminal set $T$. Similarly, the $c$-ConneCted SeParator problem is NP-complete for any $c \geq 1$, since we can transform any instance of $c$-CONNECTED STEINER into an equivalent instance of $c$-Connected Separator by making two new vertices $s$ and $t$ adjacent to each of the terminals.

Menger's Theorem provides an equivalent definition of $c$-connectivity (see [10]): a graph is $c$ connected if any two of its vertices can be joined by $c$ internally vertex-disjoint paths. Therefore, a different way of generalizing STEINER TREE would be to require the weaker condition saying that $H$ contains $c$ internally vertex-disjoint paths between any two terminals. The following lemma shows that for $c=2$ this is almost the same problem, as any minimal solution satisfying the weaker requirement satisfies the stronger requirement as well:

Lemma 1. Let $H$ be a graph and $T \subseteq V(H)$ a set of vertices such that there are two internally vertex-disjoint paths between any $t_{1}, t_{2} \in T$. If $H$ has no proper subgraph (containing $T$ ) having this property, then $H$ is 2-connected. 
Proof. Since there are two internally vertex-disjoint paths between any $t_{1}, t_{2} \in T$, all vertices of $T$ belong to the same 2-connected component $H^{\prime}$ of $H$. Suppose $H$ has no proper subgraph that contains $T$ as well as two internally vertex-disjoint paths between any two vertices of $T$. Then we must have $H^{\prime}=H$, implying that $H$ is 2-connected.

We note that for $c \geq 3$, the analog of Lemma 1 is not true. Thus the weaker requirement would result in a different problem, but we do not investigate it further in the current paper.

Our algorithm for 2-CONNECTED STEINER crucially depends on the following structural property of any minimal solution:

Lemma 2. Let $(G, T, k)$ be an instance of the 2-ConneCted Steiner problem. If $H$ is a minimal solution, then $H-T$ is a forest.

Proof. Suppose $H$ is a minimal solution. We show that every cycle in $H$ contains at least one vertex of $T$, which implies that $H-T$ is a forest. For contradiction, let $C$ be a cycle in $H$ that contains none of the terminals. We will identify an edge $e$ of $C$ such that it remains true in $H-e$ that there are two internally vertex-disjoint paths between any two terminals. Then, by Lemma $1, H-e$ has a 2 -connected subgraph which is a solution, contradicting the minimality of $H$.

Let us define a shortcut of $C$ to be a path $P$ in $H$ of length at least 2 between two vertices $a$ and $b$ of $C$, such that none of the internal vertices of $P$ are in $C$. We claim that every terminal $t \in T$ lies on some shortcut of $C$. To see this, consider an arbitrary edge $e_{1}$ in $H$ incident with $t$ and an arbitrary edge $e_{2}$ of $C$. As $H$ is 2-connected, there is a cycle in $H$ containing both $e_{1}$ and $e_{2}$, and this cycle gives us the required shortcut.

Now let $M$ be a shortest subpath of $C$ such that there is a shortcut $P^{*}$ of $C$ between the endpoints $a$ and $b$ of $M$. Let $\bar{M}$ be the other path between $a$ and $b$ on the cycle $C$. Let $a^{\prime}$ be the neighbor of $a$ on $M$ (possibly $a^{\prime}=b$ ). We claim that after removing the edge $a a^{\prime}$ from $H$, the obtained graph $H-a a^{\prime}$ still contains two internally vertex-disjoint paths between each pair of terminals.

By the well-known properties of the 2-connected components of graphs, the relation "being in the same 2-connected component" (or equivalently, the relation "there is a cycle containing both edges") defined on the edges of $H-a a^{\prime}$ is an equivalence relation. Every edge of $\bar{M}$ is in the same equivalence class of this relation: $\bar{M}$ together with $P^{*}$ forms a cycle containing all these edges. In other words, all edges of $\bar{M}$ belong to the same 2-connected component of $H-a a^{\prime}$. We claim that every terminal also belongs to this 2-connected component. Let $t \in T$. As observed above, there is a shortcut $P_{t}$ going through $t$. Let $M_{t}$ be the subpath of the cycle $C$ between the endpoints of $P_{t}$ avoiding $a a^{\prime}$. The paths $P_{t}$ and $M_{t}$ together form a cycle $C_{t}$. This cycle $C_{t}$ contains at least one edge of $\bar{M}$, since $M_{t}$ cannot be a proper subpath of $M$ by the minimality of $M$. Thus the edges of $C_{t}$, and consequently terminal $t$, are in the same 2 -connected component as the edges of $\bar{M}$. We deduce that all terminals in $T$ belong to the same 2-connected component of $H-a a^{\prime}$. Hence, there are two internally vertex-disjoint paths in $H-a a^{\prime}$ between any two terminals $t_{1}, t_{2} \in T$, yielding the desired contradiction to the assumption that $H$ is a minimal solution.

Lemma 2 tells us that we have to find an appropriate forest that connects to the terminals in an appropriate way. Fixed-parameter tractability results for finding trees (or more generally, bounded-treewidth graphs) under various technical constraints can usually be obtained using standard application of dynamic programming. A technique that has proved to be particularly useful for proving FPT results of this type is color coding, introduced by Alon, Yuster and Zwick [1]. A $k$-perfect family of hash functions is a family $\mathcal{H}$ of functions from $\{1, \ldots, n\}$ to $\{1, \ldots, k\}$ such that for each $S \subseteq\{1, \ldots, n\}$ with $|S|=k$ there exists an $h \in \mathcal{H}$ such that 
$h(s) \neq h\left(s^{\prime}\right)$ for every $s, s^{\prime} \in S, s \neq s^{\prime}$. The following result is due to Alon, Yuster and Zwick [1].

Theorem 3 ([1]). For any $n, k \in \mathbb{N}$, a $k$-perfect family of hash functions from $\{1, \ldots, n\}$ to $\{1, \ldots, k\}$ consisting of $2^{O(k)} \cdot \log n$ hash functions can be constructed in $2^{O(k)} \cdot n \log n$ time.

A combination of dynamic programming and color coding yields the following lemma. The proof is a standard application of dynamic programming on the trees, but we provide a proof for completeness.

Lemma 3. Let $F$ be a forest, $G$ an undirected graph, and $c: V(F) \times V(G) \rightarrow \mathbb{Z}^{+}$a cost function. In time $f(|V(F)|) \cdot n^{O(1)}$, one can find a mapping $\phi: V(F) \rightarrow V(G)$ such that $\phi(u) \phi(v) \in E(G)$ for every $u v \in E(F)$ and the total cost $\sum_{v \in V(F)} c(v, \phi(v))$ is minimized.

Proof. It will be convenient to consider the forest $F$ as a rooted tree where a subset $D \subseteq E(F)$ of edges are marked "special", meaning that they are not part of the forest. Let us fix an ordering of the children of each vertex. Let $F_{v}$ be the subtree rooted at vertex $v$, and let $F_{v, i}$ be the subtree consisting of $v$, the first $i$ children of $v$, and all the descendants of these $i$ children. Our approach for discovering $F$ in $G$ follows the color coding strategy, which involves coloring the graph with $|V(F)|$ colors - that is, each vertex in $G$ is assigned one element from $C=\left\{c_{1}, \ldots, c_{|V(F)|}\right\}$. A coloring is good if it assigns a distinct color to each vertex of $F$ in $G$. We describe a procedure for finding the optimal $F$ given a good coloring of $G$. By Theorem 3, there is a family of $2^{O(|V(F)|)} \cdot \log n$ colorings such that one of them is guaranteed to be good, and such a family can be constructed in time $2^{O(|V(F)|)} \cdot n \log n$. Therefore, by trying every coloring of the family, the running time of the procedure described below increases by a factor of $2^{O(|V(F)|)} \cdot \log n$.

For $x \in V(F), y \in V(G)$, and $C^{\prime} \subseteq C$, let $A\left[x, y, C^{\prime}\right]$ denote the minimum cost of a mapping $\phi$ of $F_{x}$ into $G$ such that $\phi(x)=y$ and the images of $V\left(F_{x}\right)$ use only the colors in $C^{\prime}$, each of them exactly once. Similarly, for $x \in V(F), y \in V(G), C^{\prime} \subseteq C$, and $i \in \mathbb{Z}^{+}$, let $B\left[x, y, C^{\prime}, i\right]$ denote the minimum cost of a mapping $\phi$ of $F_{x, i}$ into $G$ such that $\phi(x)=y$ and the images of $V\left(F_{x, i}\right)$ use only the colors $C^{\prime}$, each of them exactly once. If $x$ has no children or $i=0$, then these values are trivial to determine. Otherwise, if $x_{1}, \ldots, x_{\ell}$ are the children of $x$, then we can use the recurrences

$$
\begin{aligned}
& A\left[x, y, C^{\prime}\right]=B\left[x, y, C^{\prime}, \ell\right] \\
& B\left[x, y, C^{\prime}, i\right]=\min \left\{B\left[x, y, C^{\prime} \backslash C_{i}, i-1\right]+A\left[x_{i}, y_{i}, C_{i}\right] \mid C_{i} \subseteq C^{\prime}, x x_{i} \notin D \Rightarrow y y_{i} \in E(G)\right\}
\end{aligned}
$$

The first recurrence requires no explanation. In the second equation, we guess all possibilities for the set $C_{i}$ of colors used by the subtree rooted at $x_{i}$ and a suitable $y_{i}=\phi\left(x_{i}\right)$, i.e., $y_{i}$ should be adjacent to $y$, unless $x x_{i}$ happens to be in the set $D$ of "special" edges that we consider as removed.

The cost of an optimal mapping $\phi: V(F) \rightarrow V(G)$ equals $\min \{A[r, y, C] \mid y \in V(G)\}$, where $r$ is the root of the forest $F$. It is easy to modify the algorithm in such a way that it finds not only the cost of an optimal mapping $\phi$, but also the mapping itself. The algorithm clearly runs in $f(|V(F)|) \cdot n^{O(1)}$ time.

The structural observation of Lemma 2 and the algorithm of Lemma 3 allow us to establish the fixed-parameter tractability of the 2-CONNECTED STEINER problem, which could be interesting in its own right. Furthermore, it will be used as a subroutine in our FPT-algorithm for finding a 2-connected $s-t$ separator of size at most $k$.

Theorem 4. The 2-Connected Steiner problem is FPT. 
Proof. Let $(G, T, k)$ be a yes-instance of the 2-ConneCted Steiner problem and let $H$ be a minimal solution. By Lemma $2, H-T$ is a forest. We try all graphs $H$ on at most $k$ vertices that are candidates for being isomorphic to the solution $H$; that is, $H$ is 2-connected, $T \subseteq V(H)$, and $H-T$ is a forest. The number of such graphs is a function of $k$ only. For each such $H$, we define a cost function $c$ such that for $x \in V(H-T)$ and $y \in V(G)$, we have $c(x, y)=0$ if $N_{H}(x) \cap T \subseteq N_{G}(y) \cap T$ and $c(x, y)=\infty$ otherwise. In other words, we allow mapping $x$ to $y$ only if every terminal neighbor of $x$ in $H$ is also a neighbor of $y$ in $G$. Let us use the algorithm of Lemma 3 to find a mapping $\phi$ of $H-T$ into $G$ minimizing the cost. If the cost of $\phi$ is 0 , then $\phi$ can be extended to a mapping of $H$ into $G$, showing that $H$ is a subgraph of $G$, which gives us a solution. Otherwise, we proceed with the next candidate $H$. If the algorithm finds no solution after processing all candidates, we can safely return "no".

In order to prove that 2-Connected SEPARATOR is FPT, we will make use of the Treewidth Reduction Theorem due to Marx, O'Sullivan and Razgon [24, 25]. In fact, instead of using the Treewidth Reduction Theorem itself, we use a lemma (a slight reformulation of Lemma 2.11 in [25]) that forms its crucial ingredient. In order to state it, we need an additional definition. Let $G$ be a graph and $C \subseteq V(G)$. The graph torso $(G, C)$ has vertex set $C$, and vertices $a, b \in C$ are connected by an edge if $a b \in E(G)$ or if there is a path in $G$ connecting $a$ and $b$ whose internal vertices are not in $C$.

Lemma 4 ([25]). Let $s$ and $t$ be two vertices of a graph $G$, let $k$ be an integer, and let $C$ be the union of all minimal $s-t$ separators in $G$ of size at most $k$. Then there is an $f(k) \cdot(n+m)$ time algorithm that returns a set $C^{\prime} \supseteq C$ disjoint from $\{s, t\}$ such that the treewidth of $\operatorname{torso}\left(G, C^{\prime}\right)$ is at most $g(k)$.

Note that even if $G$ has a 2-connected $s-t$ separator $S$ of size at most $k, G$ might not have a minimal $s-t$ separator of size at most $k$ that is 2-connected, since 2-connectivity is not a hereditary property. However, $G$ does contain a minimal $s-t$ separator that can be extended to a 2-connected set of size at most $k$. We call a set $S^{\prime} \subseteq V(G) k$-biconnectable if there is a 2-connected set $S \subseteq V(G)$ of size at most $k$ such that $S^{\prime} \subseteq S$.

Observation 5 Let $G$ be a graph. A set $S^{\prime} \subseteq V(G)$ is $k$-biconnectable if and only if $\left(G, S^{\prime}, k\right)$ is a yes-instance of the 2-CONNECTED STEINER problem.

The set $C^{\prime}$ in Lemma 4 contains every minimal $s-t$ separator $S^{\prime}$ that is $k$-biconnectable, but there is no guarantee that $S^{\prime}$ can be extended to a 2-connected set within $C^{\prime}$. The next lemma shows that we can extend $C^{\prime}$ to a larger set $C^{\prime \prime}$ such that every $k$-biconnectable $s-t$ separator $S^{\prime} \subseteq C^{\prime}$ can be extended to a 2 -connected $s-t$ separator $S \subseteq C^{\prime \prime}$ of size at most $k$.

Lemma 5. Let $s$ and $t$ be two vertices of a graph $G$, and let $k$ be an integer. There is a set $C^{\prime \prime} \subseteq V(G)$ such that the treewidth of $\operatorname{torso}\left(G, C^{\prime \prime}\right)$ is bounded by a constant depending only on $k$ and the following holds: if $G$ has a 2-connected $s-t$ separator of size at most $k$, then $G$ also has a 2-connected $s-t$ separator $S$ of size at most $k$ such that $S \subseteq C^{\prime \prime}$. Moreover, such a set $C^{\prime \prime}$ can be found in time $h(k) \cdot n^{O(1)}$.

Proof. Let $C^{\prime} \subseteq V(G)$ be the set of Lemma 4 that contains every minimal $s-t$ separator of $G$ of size at most $k$, such that the treewidth of $\operatorname{torso}\left(G, C^{\prime}\right)$ is bounded by $g(k)$ for some function $g$ depending only on $k$. We add the vertices $s$ and $t$ to $C^{\prime}$; note that this only increases the treewidth of the graph torso $\left(G, C^{\prime}\right)$ by at most 2 , so the treewidth of this graph is still bounded by a function of $k$. Let $K_{1}, \ldots, K_{q}$ be the connected components of $G-C^{\prime}$, and let $N_{i}$ be the neighborhood of $K_{i}$ in $C^{\prime}$ for $1 \leq i \leq q$. By the definition of torso, each $N_{i}$ forms a clique in torso $\left(G, C^{\prime}\right)$. Since each clique of a graph must appear in a single bag of any tree decomposition 
of that graph, we have $\left|N_{i}\right| \leq \operatorname{tw}\left(\operatorname{torso}\left(G, C^{\prime}\right)\right)+1$, so the size of each $N_{i}$ is bounded by a function of $k$ only.

Our algorithm for constructing $C^{\prime \prime}$ iterates over all $i \in\{1, \ldots, q\}$, all non-empty subsets $X \subseteq N_{i}$, and all graphs $F_{i, X}$ on at most $k-|X|$ vertices. For each choice of $i, X$ and $F_{i, X}$, the algorithm considers each of the $2^{|X|(k-|X|)}$ graphs $G_{i, X}$ that can be obtained from $G\left[V\left(K_{i}\right) \cup X\right]$ and $F_{i, X}$ by adding a subset of edges from the set $E^{\prime} \subseteq\left\{x y \mid x \in X, y \in V\left(F_{i, X}\right)\right\}$. For each $G_{i, X}$, we run the algorithm of Theorem 4 to check if there is a solution $H_{i, X}$ for the 2-Connected Steiner problem with instance $\left(G_{i, X}, X \cup V\left(F_{i, X}\right), k\right)$. If so, we take $H_{i, X}$ to be the minimum such solution; otherwise we let $H_{i, X}=\emptyset$. For each $H_{i, X}$, we mark all the vertices of $H_{i, X}$ that belong to $K_{i}$. Finally, we define $C^{\prime \prime}$ to be the set consisting of all the vertices of $C^{\prime}$ plus all the vertices that were marked during this entire process.

In order to prove the correctness of this algorithm, let us consider a 2-connected $s-t$ separator $S$ of size at most $k$ in $G$ such that $\left|S \backslash C^{\prime \prime}\right|$ is as small as possible. We need to show that $\left|S \backslash C^{\prime \prime}\right|=0$. For contradiction, we assume that $\left|S \backslash C^{\prime \prime}\right| \geq 1$. Let $K_{i}$ be a connected component of $G-C^{\prime}$ such that $K_{i}$ contains a vertex of $S \backslash C^{\prime \prime}$, let $X=S \cap N_{i}$, and let $F_{i, X}=G\left[S \backslash\left(V\left(K_{i}\right) \cup X\right)\right]$. Note that $X \neq \emptyset$. Also note that $X \cup V\left(F_{i, X}\right)$ is a $k$-biconnectable set in the graph $G\left[V\left(K_{i}\right) \cup X \cup V\left(F_{i, X}\right)\right]$. Hence, by Observation $5,\left(G\left[V\left(K_{i}\right) \cup X \cup V\left(F_{i, X}\right)\right], X \cup\right.$ $\left.V\left(F_{i, X}\right), k\right)$ is a yes-instance of 2-Connected Steiner. Since $X \neq \emptyset$, in some iteration of the algorithm, we considered a graph $G_{i, X}$ that is isomorphic to $G\left[V\left(K_{i}\right) \cup X \cup V\left(F_{i, X}\right)\right]$ and hence found a minimum solution $H_{i, X}$ of 2-CONNECTED Steiner for exactly the instance $\left(G\left[V\left(K_{i}\right) \cup X \cup V\left(F_{i, X}\right)\right], X \cup V\left(F_{i, X}\right), k\right)$. Let $S^{\prime}=V\left(H_{i, X}\right) \cup X \cup V\left(F_{i, X}\right)$. By construction, $S^{\prime}$ is 2-connected. Note that $S \cap C^{\prime}$ is an $s-t$ separator, since otherwise there would be a minimal $s-t$ separator of size at most $k$ in $G$ that contains a vertex outside $C^{\prime}$, contradicting Lemma 4. Since $S \cap C^{\prime} \subseteq S^{\prime}$, the set $S^{\prime}$ is an $s-t$ separator. It is clear that $S^{\prime} \subseteq C^{\prime \prime}$, which means that $\left|S^{\prime} \backslash C^{\prime \prime}\right|=0$. Hence $\left|S^{\prime} \backslash C^{\prime \prime}\right|<\left|S \backslash C^{\prime \prime}\right|$, contradicting the minimality of $S$.

For each $i \in\{1, \ldots, q\}$, we test at most $\left|N_{i}\right|^{k}$ sets $X \subseteq N_{i}$, and for each of these sets $X$, we test at most $2^{k^{2}}$ different graphs $G_{i, X}$. For each graph $G_{i, X}$, we mark at most $k$ vertices of $K_{i}$, so the total number of vertices in $K_{i}$ that are marked is at most $k \cdot 2^{k^{2}} \cdot\left|N_{i}\right|^{k}$. Recall that the size of each $N_{i}$ is bounded by a function of $k$ only, which implies that the same holds for $\left|C^{\prime \prime} \cap V\left(K_{i}\right)\right|$ and consequently for the treewidth of torso $\left(K_{i}, C^{\prime \prime} \cap V\left(K_{i}\right)\right)$. It follows that the difference between the treewidth of $\operatorname{torso}\left(G, C^{\prime \prime}\right)$ and the treewidth of $\operatorname{torso}\left(G, C^{\prime}\right)$ is a constant depending on $k$ (see also Lemma 2.9 in [25]), implying that the treewidth of $\operatorname{torso}\left(G, C^{\prime \prime}\right.$ ) is bounded by a function of $k$. Finding the set $C^{\prime}$ can be done in time $f(k) \cdot(m+n)$ by Lemma 4. For each choice of $i$, the possible number of different graphs $G_{i, X}$, and consequently the number of instances of 2-CONNECTED STEINER we have to solve, is at most $k \cdot 2^{k^{2}} \cdot\left|N_{i}\right|^{k}$. Since 2-ConneCted Steiner is FPT by Theorem 4, the overall running time of the algorithm is $h(k) \cdot n^{O(1)}$ for some function $h$ that depends only on $k$.

Theorem 6. The 2-Connected Separator problem is FPT.

Proof. Let $(G, s, t, k)$ be an instance of 2-Connected Separator. We start by constructing the set $C^{\prime \prime} \subseteq V(G)$ of Lemma 4 . Let $G^{*}=\operatorname{torso}\left(G, C^{\prime \prime}\right)$. We assign a color to each edge $u v$ in $G^{*}$ : we color $u v$ black if $u v$ is also an edge in $G$, and we color $u v$ red otherwise. By Lemma 5 , $G$ contains a 2-connected $s-t$ separator $S$ of size at most $k$ if and only if $G^{*}$ contains an $s-t$ separator $S^{*}$ of size at most $k$ such that deleting the red edges from $G^{*}\left[S^{*}\right]$ results in a 2connected graph. The theorem now follows from Courcelle's Theorem [8] and the fact that this problem can be expressed in monadic second-order logic (see [25]).

We now show that the $c$-Connected Steiner problem becomes hard when the connectivity of the solution is required to be at least 3. In the proof of Theorem 7 below, as well as 
in the hardness proofs in the next section, we give parameterized reductions from the MULTICOlORED Clique problem. This problem takes as input a graph $G$, an integer $k$, and a partition of $V(G)$ into $k$ independent sets $C_{1}, \ldots, C_{k}$ called color classes. The objective is to decide whether there exists a subset $S \subseteq V(G)$ such that $|S|=k, G[S]$ is a complete graph, and $\left|S \cap C_{i}\right|=1$ for all $1 \leq i \leq k$; such a set $S$ is called a multicolored clique. This problem is known to be $W[1]$-hard when parameterized by $k$ [14].

Theorem 7. c-Connected Steiner is $W[1]$-hard for any $c \geq 3$.

Proof. We first show that the theorem holds for $c=3$. We reduce from Multicolored Clique. Note that Multicolored Clique remains $W$ [1]-hard when we assume that $k \geq 3$, and we make this assumption in the proof below. Let $(G, k)$ be an instance of Multicolored Clique with color classes $C_{1}, \ldots, C_{k}$. Starting from $G$, we construct a graph $G^{\prime}$ as follows. We first subdivide every edge $u v \in E(G)$ exactly once by adding a vertex $x_{u v}$, deleting the edge $u v$, and adding the edges $u x_{u v}, v x_{u v}$. We then add a number of terminals as follows. For every $1 \leq i<j \leq k$, we add a terminal $g_{i, j}$ and make it adjacent to all vertices $x_{u v}$ with $u \in C_{i}$ and $v \in C_{j}$. For every $1 \leq i \leq k$, we add a terminal $g_{i}$ and make it adjacent to all vertices in $C_{i}$. We then add two terminals $a_{1}, a_{2}$ and make both them adjacent to $g_{i}$ for every $1 \leq i \leq k$. We also add two terminals $b_{1}, b_{2}$ and make both of them adjacent to $g_{i, j}$ for every $1 \leq i<j \leq k$. This finishes the construction of the graph $G^{\prime}$. We define $T$ to be the set of all terminals in $G^{\prime}$, and we define $k^{\prime}=2 k+k(k-1)+4$. We show that $(G, k)$ is a yes-instance of Multicolored Clique if and only if $\left(G^{\prime}, T, k^{\prime}\right)$ is a yes-instance of 3-Connected Steiner.

First suppose that $G$ has a multicolored clique $X$, and let $c_{i}=X \cap C_{i}$ for every $1 \leq i \leq k$. Let $S \subseteq V\left(G^{\prime}\right)$ be the set containing each of the $k$ vertices in $X$, each of the $k(k-1) / 2$ vertices in the set $\left\{x_{c_{i} c_{j}} \mid c_{i}, c_{j} \in X\right\}$, and each of the $k+k(k-1) / 2+4$ terminals in $G^{\prime}$. We claim that $G^{\prime}[S]$ is a 3 -connected subgraph of $G^{\prime}$ on at most $k^{\prime}$ vertices that contains all the terminals. Note that $|S|=2 k+k(k-1)+4=k^{\prime}$. It remains to show that $G^{\prime}[S]$ is 3-connected. For convenience, we define $A=\left\{a_{1}, a_{2}\right\} \cup\left\{g_{i} \mid 1 \leq i \leq k\right\}, B=\left\{b_{1}, b_{2}\right\} \cup\left\{g_{i, j} \mid 1 \leq i<j \leq k\right\}$ and $C=S \backslash(A \cup B)=X \cup\left\{x_{c_{i} c_{j}} \mid c_{i}, c_{j} \in X\right\}$. Recall that we may assume the number of color classes $C_{i}$ to be at least 3 . Hence there is a matching $M_{A} \subseteq E\left(G^{\prime}\right)$ containing at least three edges with one endpoint in $A$ and one endpoint in $C$, as well as a matching $M_{B} \subseteq E\left(G^{\prime}\right)$ with at least three edges between $B$ and $C$. In order to show that $S$ induces a 3 -connected subgraph in $G^{\prime}$, it suffices to show that for every pair of distinct vertices $s, s^{\prime} \in S$, the graph $G^{\prime}[S]-\left\{s, s^{\prime}\right\}$ is connected. This clearly is the case if $\left\{s, s^{\prime}\right\}=\left\{a_{1}, a_{2}\right\}$ and if $\left\{s, s^{\prime}\right\}=\left\{b_{1}, b_{2}\right\}$. Suppose that $\left\{s, s^{\prime}\right\} \neq\left\{a_{1}, a_{2}\right\}$ and $\left\{s, s^{\prime}\right\} \neq\left\{b_{1}, b_{2}\right\}$. Then each of the graphs $G_{A}^{\prime}=G^{\prime}\left[A \backslash\left\{s, s^{\prime}\right\}\right]$, $G_{B}^{\prime}=G^{\prime}\left[B \backslash\left\{s, s^{\prime}\right\}\right]$ and $G_{C}^{\prime}=G^{\prime}\left[C \backslash\left\{s, s^{\prime}\right\}\right]$ is connected. Moreover, due to the existence of the matchings $M_{A}$ and $M_{B}$ in $G^{\prime}$, there is at least one edge between $G_{A}^{\prime}$ and $G_{C}^{\prime}$ and at least one edge between $G_{B}^{\prime}$ and $G_{C}^{\prime}$. This implies that the graph $G^{\prime}[S]-\left\{s, s^{\prime}\right\}$ is connected.

For the reverse direction, suppose $G^{\prime}$ has a 3-connected subgraph $G^{\prime}[S]$ on at most $k^{\prime}$ vertices that contains all the terminals. The 3-connectedness of $G^{\prime}[S]$ implies that every vertex in $S$ has degree at least 3 in $G^{\prime}[S]$. Hence each of the terminals, apart from $a_{1}, a_{2}, b_{1}, b_{2}$, must have at least one neighbor in $S$ that is not a terminal. Recall that $|S| \leq k^{\prime}=2 k+k(k-1)+4$, and that there are $k+k(k-1) / 2+4$ terminals in $G^{\prime}$. Hence $S$ contains at most $k+k(k-1) / 2$ vertices that are not terminals. This implies that each terminal, apart from $a_{1}, a_{2}, b_{1}, b_{2}$, has exactly one neighbor in $S$ that is not a terminal. For each of the terminals $g_{i}$, let $c_{i}$ be this neighbor, and for each $g_{i, j}$, let $x_{i, j}$ be this neighbor. $S$ contains no other vertices than the ones mentioned above. Since every vertex in $S$ must have degree at least 3 in $G^{\prime}[S]$ and each of the vertices $x_{i, j}$ has degree exactly 3 in $G^{\prime}$, we know that both non-terminal neighbors of $x_{i, j}$ in $G^{\prime}$ belong to $S$, for every $1 \leq i<j \leq k$. This means that, for every $1 \leq i<j \leq k$, vertex $x_{i, j}$ is adjacent to $c_{i}$ and $c_{j}$. We conclude that the vertices $\left\{c_{1}, \ldots, c_{k}\right\}$ form a multicolored clique in $G$. 
We can easily modify the above reduction in order to show that $c$-CONNECTED STEINER is $W[1]$-hard for any fixed $c \geq 4$. After constructing $G^{\prime}$ as described in the reduction for $c=3$, we simply add a clique of $c-3$ additional terminals to $G^{\prime}$, make each of them universal by making them adjacent to all vertices in $G^{\prime}$, and increase the parameter $k^{\prime}$ by $c-3$. The result follows from the observation that the connectivity of a graph increases by exactly 1 each time a universal vertex is added to the graph.

Since we can transform an instance of $c$-CONnECTED STEINER into an equivalent instance of $c$-ConneCTED SEPARATOR by making two new vertices $s$ and $t$ adjacent to each of the terminals, Theorem 7 readily implies the following result.

Theorem 8. c-ConneCted SEPARATOR is $W[1]$-hard for any $c \geq 3$.

\section{More $W[1]$-Hardness Results on General Graphs}

We say that a graph $G$ is $r$-regular if the degree of every vertex in $G$ is exactly $r$. For every $r \geq 0$, let $r$-Regular Separator denote the problem of deciding whether an input graph $G$ has an $s-t$ separator $S$ of size at most $k$ such that $G[S]$ is $r$-regular. Since the class of 0-regular graphs is hereditary, Theorem 1 implies that 0-REgular SEPARATOR, i.e., the problem of finding an $s-t$ separator that is an independent set of size at most $k$, is FPT. We show that $r$-REgulaR SEPARATOR is $W[1]$-hard for every $r \geq 1$. Note that the class of $r$-regular graphs is not hereditary for any $r \geq 1$.

Theorem 9. $r$-Regular Separator is $W[1]$-hard for any $r \geq 1$.

Proof. Let $r \geq 1$ be an integer. We show that $r$-Regular SEPARATOR is $W[1]$-hard by a reduction from Multicolored Clique. Let $(G, k)$ be an instance of Multicolored Clique with color classes $C_{1}, \ldots, C_{k}$. We create a graph $G^{\prime}$ as follows. We start with a copy of the complement of $G$. For every color class $C_{i}$, we add a clique $X_{i}$ on $r$ vertices, and we make each vertex in $X_{i}$ adjacent to every vertex in $C_{i}$. Let $X=\bigcup_{i=1}^{k} X_{i}$. Finally, we add two vertices $s$ and $t$, and make both these vertices adjacent to every vertex in $X$. We show that $(G, k)$ is a yes-instance of Multicolored Clique if and only if $G^{\prime}$ has an $r$-regular $s-t$ separator of size at most $(r+1) k$.

Suppose $G$ had a multicolored clique $C=\left\{c_{1}, \ldots, c_{k}\right\}$, where $c_{i} \in C_{i}$ for every $1 \leq i \leq k$. Note that the set $C$ forms an independent set in $G^{\prime}$. Consider the set $C \cup X$. Since every path from $s$ to $t$ in $G^{\prime}$ contains a vertex of $X$, the set $C \cup X$ separates $s$ from $t$. Moreover, $C \cup X$ has size $(r+1) k$ and induces an $r$-regular subgraph in $G^{\prime}$. For the reverse direction, suppose there exists an $s-t$ separator $S$ of size at most $(r+1) k$ in $G^{\prime}$ such that $G^{\prime}[S]$ is $r$-regular. Every vertex in $X$ is adjacent to both $s$ and $t$, so we must have $X \subseteq S$. Since $G^{\prime}[S]$ is $r$-regular and every vertex in $X$ has degree $r-1$ in $G^{\prime}[X]$ by construction, every vertex $x_{i} \in X$ has exactly one neighbor $c_{i}$ in $S \backslash X$, which means that $c_{i} \in C_{i}$. Moreover, the vertices $\left\{c_{1}, \ldots, c_{k}\right\}$ must form an independent set in $G^{\prime}$, implying that $\left\{c_{1}, \ldots, c_{k}\right\}$ is a multicolored clique in $G$.

The diameter of a graph $G$ is the maximum distance between any two vertices in $G$, where the distance between two vertices $u$ and $v$ is defined as the number of edges in a shortest path from $u$ to $v$. As mentioned earlier, the problem of finding an $s-t$ separator that forms a clique is well-known to be solvable in polynomial time [28]. Since cliques induce subgraphs of diameter 1 , it is natural to consider the problem of finding an $s-t$ separator that induces a subgraph of diameter 2 , or, more generally, of any fixed diameter $d \geq 2$. Note that for any $d \geq 2$, the class of graphs with diameter $d$ is not hereditary; consider for example a chordless cycle on $2 d+1$ vertices. The class of graphs with diameter 1 , however, is hereditary. 


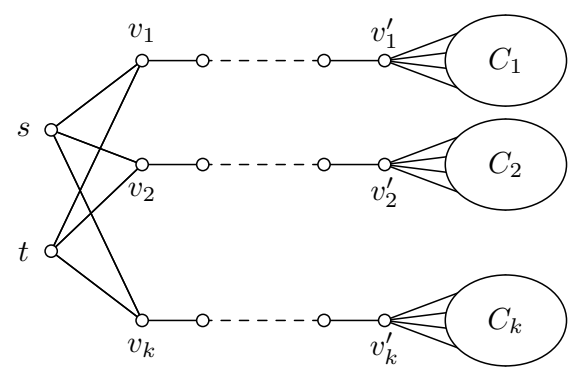

Fig. 1. The graph $H$ constructed from an instance $(G, k)$ of Multicolored Clique, as described in the case $d \geq 3$ in the proof of Theorem 10. Edges between color classes have note been drawn.

The $d$-Diameter Separator problem is to decide if an input graph $G$ has an $s$ - $t$ separator $S$ of size at most $k$ such that $G[S]$ has diameter $d$. We now show that $d$-Diameter Separator is $W[1]$-hard for any $d \geq 2$. The reductions in the proof of Theorem 10 below also show that the problem of finding an $s-t$ separator $S$ of size at most $k$ such that $G[S]$ has diameter at most $d$ is $W[1]$-hard for any $d \geq 2$.

Theorem 10. $d$-Diameter SePARAtor is $W[1]$-hard for any $d \geq 2$.

Proof. In order to keep the reductions as clear as possible, we first consider the case $d \geq 3$, after which we deal with the slightly trickier case $d=2$.

Case 1: $d \geq 3$.

Let $(G, k)$ be an instance of the Multicolored Clique problem with color classes $C_{1}, \ldots, C_{k}$. For any fixed $d \geq 3$, we construct an instance $(H, l)$ of $d$-Diameter SEParator from $(G, k)$ in the way described below, where we distinguish between odd and even values of $d$.

If $d=2 p+1$ for some $p \geq 1$, we create the graph $H$ as follows. Start with a copy of $G$, and add two vertices $s$ and $t$. For every $1 \leq i \leq k$, add a path $P_{i}$ on $p$ vertices. Let $v_{i}$ and $v_{i}^{\prime}$ be the degree 1 vertices of $P_{i}\left(v_{i}=v_{i}^{\prime}\right.$ if $\left.p=1\right)$. Make $v_{i}$ adjacent to both $s$ and $t$, and make $v_{i}^{\prime}$ adjacent to every vertex in $C_{i}$. Finally, we set $l=(p+1) k$. See Figure 1 for a schematic representation of the graph $H$; for clarity, edges between the color classes have not been drawn. We show that $G$ has a multicolored clique of size $k$ if and only if $H$ has an $s$ - $t$ separator of size $l$ that induces a subgraph of diameter $d$ in $H$.

Suppose $G$ has a multicolored clique $X=\left\{c_{1}, \ldots, c_{k}\right\}$, where $c_{i} \in C_{i}$ for every $1 \leq i \leq k$. Let $S$ be the set containing the vertices of $X$, as well as the vertices of every path $P_{i}$. Since $S$ contains each of the vertices $v_{i}$, and those vertices are the only neighbors of $s$ and $t$ in $H, S$ clearly is an $s-t$ separator. For any $1 \leq i<j \leq k$, the unique shortest path between $v_{i}$ and $v_{j}$ in $H[S]$ uses all the vertices of the paths $P_{i}$ and $P_{j}$, as well as the vertices $c_{i}$ and $c_{j}$. Hence the distance between $v_{i}$ and $v_{j}$ in $H[S]$ is $2 p+1$. For every other pair of vertices in $S$, the distance in $H[S]$ is smaller. Hence $S$ induces a subgraph of diameter $2 p+1=d$ in $H$. Finally, the size of $S$ is $(p+1) k=l$, since it contains $p$ vertices for each of the $k$ paths $P_{i}$, as well as each of the $k$ vertices of $X$.

For the reverse direction, suppose that there exists an $s-t$ separator $S$ of size at most $l$ such that $H[S]$ has diameter $d$. Note that any $s-t$ separator must contain each of the vertices $v_{i}$, since these vertices are adjacent to both $s$ an $t$. Hence $S$ contains each of the vertices $v_{i}$. Since $H[S]$ has diameter $d$, we know that, in particular, $H[S]$ is connected. By construction, this implies that $S$ must contain all the vertices of each of the paths $P_{i}$, which amounts to $p k$ vertices in total. It also implies that $S \cap C_{i} \neq \emptyset$ for every $1 \leq i \leq k$. The assumption that $S$ 
has size $l=(p+1) k$ implies that $S$ contains exactly one vertex from each of the color classes $C_{i}$. For every $1 \leq i \leq k$, let $c_{i}$ be the only vertex in $S \cap C_{i}$, and let $X=\left\{c_{1}, \ldots, c_{k}\right\}$. Suppose that $X$ has two vertices $c_{i}$ and $c_{j}$ that are not adjacent. Then the distance between $v_{i}$ and $v_{j}$ is greater than $2 p+1$ in $H[S]$, contradicting the assumption that $H[S]$ has diameter $d$. Hence the vertices of $X$ form a multicolored clique in $G$.

If $d=2 p$ for some $p \geq 2$, then we create $H$ as we did for odd values of $d$, with two small modifications: each path $P_{i}$ has $p-1$ vertices instead of $p$ vertices (and now $v_{i}=v_{i}^{\prime}$ if $p=2$ ), and every edge between any two color classes, i.e., every edge of the copy of $G$ in $H$, is subdivided exactly once. We set $l=p k+\left(\begin{array}{c}k \\ 2\end{array}\right)$.

Suppose $G$ has a multicolored clique $X=\left\{c_{1}, \ldots, c_{k}\right\}$, where $c_{i} \in C_{i}$ for every $1 \leq i \leq k$. For every two vertices $c_{i}$ and $c_{j}$ in $X$, let $y_{i j}$ be the vertex in $H$ that subdivided the edge $c_{i} c_{j}$, and let $Y$ be the set containing all $\left(\begin{array}{c}k \\ 2\end{array}\right)$ of these vertices $y_{i j}$. We define $S$ to be the set containing the vertices of $X \cup Y$, as well as the vertices of every path $P_{i}$. It is clear that $S$ is an $s^{-} t$ separator. For any $1 \leq i<j \leq k$, the unique shortest path between $v_{i}$ and $v_{j}$ in $H[S]$ uses all the vertices of the paths $P_{i}$ and $P_{j}$, as well as the vertices $c_{i}, x_{i j}$, and $c_{j}$. Hence the distance between $v_{i}$ and $v_{j}$ in $H[S]$ is $2 p$. For every other pair of vertices in $S$, the distance in $H[S]$ is smaller. Hence $S$ induces a subgraph of diameter $2 p=d$ in $H$. Finally, the size of $S$ is $(p+1) k=l$, since it contains $p-1$ vertices for each of the $k$ paths $P_{i}$, as well as each of the $k$ vertices of $X$, and each of the $\left(\begin{array}{c}k \\ 2\end{array}\right)$ vertices of $Y$.

Now suppose that there exists an $s-t$ separator $S$ of size at most $l$ such that $H[S]$ has diameter $d$. Similar to the case for odd $d$, we know that $S$ contains all the vertices of each of the paths $P_{i}$, which amounts to $(p-1) k$ vertices in total. We also know that $S \cap C_{i} \neq \emptyset$ for every $1 \leq i \leq k$. This leaves a budget of at most $\left(\begin{array}{c}k \\ 2\end{array}\right)$ vertices. The assumption that $H[S]$ has diameter $d$ and the restrictions imposed by the budget imply that $S$ contains exactly one vertex, say $c_{i}$, from every color class $C_{i}$, as well as the vertex $y_{i j}$ for every two vertices $c_{i}$ and $c_{j}$ belonging to $S$. By the construction of $H$, it follows that the vertices $\left\{c_{1}, \ldots, c_{k}\right\}$ form a clique in $G$.

Case 2: $d=2$.

Let $(G, k)$ be an instance of the Multicolored Clique problem with color classes $C_{1}, \ldots, C_{k}$. We construct an instance $(H, l)$ of Diameter-2 Separator from $(G, k)$ as follows (see also Figure 2, where a schematic drawing of the graph $H$ is given). We start with a copy of $G$. For every color class $C_{i}$, we introduce a pair of vertices $u_{i}$ and $v_{i}$, and for every pair of color classes $C_{i}$ and $C_{j}$, we introduce four vertices $w_{i j}, x_{i j}, y_{i j}$ and $z_{i j}$. Finally, we introduce vertices $s, t$ and $g$. For every $1 \leq i \leq k$, we make the vertices $u_{i}$ and $v_{i}$ adjacent to all the vertices in $C_{i}$, and $g$ is made adjacent to all the vertices in every color class. We add an edge between each pair of vertices in the set $C=\left\{w_{i j}, x_{i j}, y_{i j}, z_{i j} \mid 1 \leq i, j \leq k\right\} \cup\{g\}$, i.e., we make $C$ into a clique. The vertices $u_{i}, v_{i}$, and all the vertices in $C$ are made adjacent to both $s$ and $t$. Finally, for all $1 \leq i<j \leq k$, we introduce the edges $w_{i j} u_{i}, w_{i j} u_{j}, x_{i j} u_{i}, x_{i j} v_{j}$, and $y_{i j} v_{i}, y_{i j} u_{j}, z_{i j} v_{i}, z_{i j} v_{j}$. To complete the construction, we set $l=3 k+4\left(\begin{array}{c}k \\ 2\end{array}\right)+1$. We now show that $G$ has a multicolored clique of size $k$ if and only if there exists an $s-t$ separator of size $l$ that induces a subgraph of diameter 2 in $H$.

Suppose $G$ has a multicolored clique $X=\left\{c_{1}, \ldots, c_{k}\right\}$, where $c_{i} \in C_{i}$ for every $1 \leq i \leq k$. Notice that the vertices of $\left\{u_{i}, v_{i} \mid 1 \leq i \leq k\right\} \cup C$ form an $s-t$ separator. To complete this set into an $s-t$ separator of diameter 2 , we include $g$ and the vertices of $X$. Let $S$ denote the $s-t$ separator thus obtained. Let $u$ and $v$ be two distinct vertices in $S$. The following case analysis shows that there is either an edge between $u$ and $v$, or they have a common neighbor in $S$.

- If $u \in C$ and $v \in C$, then $(u, v) \in E(H)$ since $C$ is a clique.

- If $u \in X$ and $v \in X$, then $(u, v) \in E(H)$ since $X$ is a multicolored clique. 


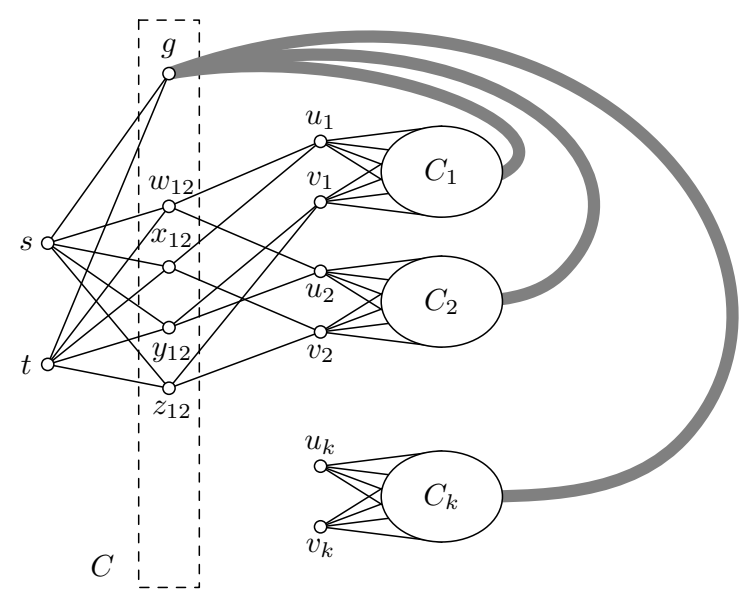

Fig. 2. The graph $H$ constructed from an instance $(G, k)$ of Multicolored Clique, as described in the case $d=2$ in the proof of Theorem 10. Many vertices and edges have been omitted for clarity; the bold edges indicate that $g$ is adjacent to all the vertices in every color class.

- If $u \in C$ and $v \in X$, then $g$ is a common neighbor of $u$ and $v$.

- If $u=u_{i}$ and $v=v_{i}$, then $c_{i}$ is a common neighbor of $u$ and $v$.

- If $u \in C$ and $v \in\left\{u_{i}, v_{i}\right\}$, then either $u$ and $v$ are adjacent, or, for any $j \neq i$, one of the vertices $w_{i j}, x_{i j}, y_{i j}, z_{i j}$ is a common neighbor of $u$ and $v$.

- If $u \in\left\{u_{i}, v_{i}\right\}$ and $v \in\left\{u_{j}, v_{j}\right\}$, then one of the vertices $w_{i j}, x_{i j}, y_{i j}, z_{i j}$ is a common neighbor of $u$ and $v$.

- If $u=c_{i}$ and $v \in\left\{u_{j}, v_{j}\right\}$, then $c_{j}$ is a common neighbor of $u$ and $v$.

Hence $G[S]$ has diameter 2 . Notice that $S$ has size $3 k+4\left(\begin{array}{c}k \\ 2\end{array}\right)+1=l$.

For the reverse direction, suppose $S$ is an $s-t$ separator of size $l$ such that $H[S]$ has diameter 2. Notice that $\left\{u_{i}, v_{i} \mid 1 \leq i \leq k\right\} \cup C \subseteq S$, since each of these vertices is adjacent to both $s$ and $t$. Notice further that $\left|\left\{u_{i}, v_{i} \mid 1 \leq i \leq k\right\} \cup C\right|=2 k+4\left(\begin{array}{c}k \\ 2\end{array}\right)+1$. Since $S$ is a separator of size $l$, this means that we have a budget of $k$ vertices remaining. The graph $H[S]$ has diameter 2, so $u_{i}$ and $v_{i}$ have a common neighbor in $S$, and consequently $S \cap C_{i} \neq \emptyset$, for every $1 \leq i \leq k$. The fact that the remaining budget is $k$ implies that contains exactly one vertex from each color class $C_{i}$. Let $c_{i}$ denote the vertex in $S \cap C_{i}$, and let $X=\left\{c_{1}, \ldots, c_{k}\right\}$. We now argue that $X$ forms a clique in $G$. For contradiction, suppose there exists a pair $(i, j)$ such that $c_{i} c_{j} \notin E(H)$. All neighbors of $v_{j}$ in the separator $S$ belong to the set $C \cup\left\{c_{j}\right\}$, and $c_{i}$ is not adjacent to any of those vertices. Note, in particular, that $c_{i}$ is adjacent to $g$, but $v_{j}$ is not. Hence $c_{i}$ and $v_{j}$ have no common neighbor in $S$, contradicting our assumption that $S$ is an $s-t$ separator inducing a subgraph with diameter 2 . We conclude that $C$ is a multicolored clique in $G$, which completes the proof of the theorem.

\section{Finding $s-t$ Separators in Graphs of Bounded Degree}

Theorem 1 states that $\mathcal{G}$-SEPARATOR is FPT for any decidable and hereditary graph class $\mathcal{G}$. In the previous sections, we identified several non-hereditary graph classes $\mathcal{G}$ for which $\mathcal{G}$ SEPARATOR is $W[1]$-hard on general graphs. In this section, we prove that for any decidable (but not necessarily hereditary) graph class $\mathcal{G}$, the $\mathcal{G}$-SEPARATOR problem is FPT parameterized jointly by the size of the separator and the maximum degree. We point out that there are 
several graph classes $\mathcal{G}$ for which $\mathcal{G}$-SePARATOR is NP-complete on graphs of bounded degree; the class of connected graphs is just one example (see Theorem 15 in Section 5).

In order to obtain the main result of this section, we study the following problem:

PATtern SEPARATOR
Input: Two graphs $G$ and $H$, and two vertices $s$ and $t$ of $G$.
Question: Does $G$ have an $s^{-} t$ separator $S$ such that $G[S]$ is isomorphic to
$H ?$

We show that PATtern SEPARATOR is FPT parameterized jointly by the number of vertices in the "pattern" graph $H$ and the maximum degree of $G$. Note that this is obviously the case if $H$ is connected, as any vertex in a graph with maximum degree $\Delta$ is contained in at most $\Delta^{|V(H)|}$ connected vertex subsets of size $|V(H)|$. The situation is less obvious when we allow $H$ to be disconnected. We will use a variant of the color coding technique of Alon, Yuster and Zwick [1] to reduce the PATtern SeParator problem to the problem of finding an $s-t$ separator that has a certain hereditary property, which enables us to invoke Theorem 1.

For the remainder of this section, let $G$ and $H$ be two graphs, let $s$ and $t$ be two vertices of $G$, and let $H_{1}, \ldots, H_{c}$ be the connected components of $H$. We use $n$ and $m$ to denote the number of vertices and edges in $G$, respectively. Let $\psi$ be a (not necessarily proper) coloring of $G$. A subset of vertices $V^{\prime} \subseteq V(G)$ is colorful if $\psi$ colors no two vertices of $V^{\prime}$ with the same color. For any subset $C^{\prime}$ of colors, we say that $V^{\prime} \subseteq V(G)$ is $C^{\prime}$-colorful if $\left|V^{\prime}\right|=\left|C^{\prime}\right|$ and every vertex in $V^{\prime}$ receives a different color from $C^{\prime}$.

Definition 1. Let $\psi: V(G) \rightarrow\{1,2, \ldots, c, c+1\}$ be a $(c+1)$-coloring of $G$. We say that $\psi$ is $H$-good if $G$ has an $s-t$ separator $S$ satisfying the following properties:

(i) each connected component of $G[S]$ is colored monochromatically with a color from $\{1, \ldots, c\}$;

(ii) no two connected components of $G[S]$ receive the same color;

(iii) the connected component of $G[S]$ with color $i$ is isomorphic to $H_{i}$;

(iv) every vertex in $N_{G}(S)$ receives color $c+1$.

It immediately follows from Definition 1 that $(G, H, s, t)$ is a yes-instance of PATTERN SEPARATOR if and only if $G$ has an $H$-good coloring. The main idea of our algorithm is that finding a separator $S$ satisfying these requirements essentially boils down to finding a separator that is a colorful independent set, which is fixed-parameter tractable by the results of [24,25]. The following lemma plays a crucial role in our algorithm for PATTERn SEPARATOR.

Lemma 6. Given a $(c+1)$-coloring $\psi$ of $G$, we can decide in $g(|V(H)|) \cdot(n+m)$ time whether $\psi$ is $H$-good.

Proof. We describe an algorithm for deciding whether a given $(c+1)$-coloring $\psi$ of $G$ is $H$ good. Let $B$ be the set of vertices in $G$ with color $c+1$. For every connected component $X$ of the graph $G-B$ that is not monochromatic, we recolor all the vertices of $V(X)$ with color $c+1$ in $G$, and set $B=B \cup V(X)$. After this step, every connected component of $G-B$ is monochromatic. For every $1 \leq i \leq c$, let $G_{i}$ be the subgraph of $G$ induced by the vertices with color $i$. For every value of $i$ from 1 up to $c$, we check, for each connected component $X$ of the graph $G_{i}$, whether $X$ is isomorphic to $H_{i}$. If not, we color all the vertices of $V(X)$ with color $c+1$ in $G$, and set $G_{i}=G_{i}-V(X)$. If at any point we find that $G_{i}$ does not have any vertices, then $\psi$ cannot satisfy condition (iii) in Definition 1 , so we output "no". Suppose that, after this step, each connected component of $G_{i}$ is isomorphic to $H_{i}$, for every $1 \leq i \leq c$. We then repeatedly contract edges with both endpoints in the same subgraph $G_{i}$, until we obtain a 
graph $G^{\prime}$ in which the vertices with colors $1, \ldots, c$ form an independent set. Also note that $G^{\prime}$ contains at least one $\{1, \ldots, c\}$-colorful independent set $S^{*}$, as otherwise the algorithm would have returned "no" already.

Claim 1: Coloring $\psi$ is $H$-good if and only if $G^{\prime}$ has an $s$-t separator $S^{\prime}$ such that $S^{\prime}$ is a $\{1, \ldots, c\}$-colorful independent set.

We prove Claim 1 as follows. Suppose $\psi$ is $H$-good, and let $S$ be an $s^{-} t$ separator of $G$ satisfying the four properties in Definition 1. Due to properties (i) and (iv) in Definition 1, each of the connected components of the subgraph $G[S]$ was contracted to a single vertex when the graph $G^{\prime}$ was obtained from $G$. For $1 \leq i \leq c$, let $s_{i}$ be the vertex in $G^{\prime}$ that corresponds to the connected component of $G[S]$ that is isomorphic to $H_{i}$. Due to properties (i) and (ii), the set $S^{\prime}=\left\{s_{1}, \ldots, s_{c}\right\}$ is $\{1, \ldots, c\}$-colorful, and property (iv) ensures that $S^{\prime}$ is an independent set in $G^{\prime}$. Since $S$ is an $s-t$ separator in $G$, every path from $s$ to $t$ in $G$ contains a vertex of $S$. We only contracted edges in $G$ to obtain $G^{\prime}$, so every path from $s$ to $t$ in $G^{\prime}$ contains a vertex of $S^{\prime}$, implying that $S^{\prime}$ is an $s-t$ separator in $G^{\prime}$.

For the reverse direction, suppose $G^{\prime}$ has an $s^{-} t$ separator $S^{\prime}$ such that $S^{\prime}$ is a $\{1, \ldots, c\}$ colorful independent set. Consider the unique vertex $s_{i} \in S^{\prime}$ with color $i$. By the construction of $G^{\prime}, s_{i}$ corresponds to a connected component of the subgraph $G_{i}$ in $G$, and such a component is isomorphic to $H_{i}$; thus property (iii) is satisfied. Property (ii) follows from the assumption that $S^{\prime}$ is colorful. Recall that every connected component of the subgraph $G-B$ that contained more than one color from $1, \ldots, c$ was recolored with color $c+1$ in the very first step of the algorithm. Since after that step we only contracted edges in $G$ to obtain $G^{\prime}$, property (i) is satisfied. Recall that all the vertices in $G^{\prime}$ with a color in $\{1, \ldots, c\}$ form an independent set. Hence for each of those vertices, and the ones in $S^{\prime}$ in particular, all neighbors have color $c+1$. This means that every vertex in $N_{G}(S)$ must have had color $c+1$ as well, implying property (iv). This finishes the proof of Claim 1.

The graph $G^{\prime}$ can be obtained from $G$ in $O(n+m)$ time. Due to the above claim, it remains to show that we can decide in $g(|V(H)|) \cdot(n+m)$ time whether the graph $G^{\prime}$ has an $s-t$ separator $S^{\prime}$ such that $S^{\prime}$ is a $\{1, \ldots, c\}$-colorful independent set. We call such a set $S^{\prime}$ a solution below. Note that the property "being a $\{1, \ldots, c\}$-colorful independent set" is not hereditary, since only sets of cardinality $c$ can have this property. The next claim shows that we can reduce the problem of finding a solution in $G^{\prime}$ to the problem of finding a small $s-t$ separator that does satisfy a certain hereditary property.

Claim 2: Graph $G^{\prime}$ has a solution if and only if $G^{\prime}$ has an $s-t$ separator $S^{\prime \prime}$ of size at most $c$ such that $S^{\prime \prime}$ is an independent set that is $C^{\prime}$-colorful for some $C^{\prime} \subseteq\{1, \ldots, c\}$.

We prove Claim 2 as follows. If $G^{\prime}$ has an $s-t$ separator $S^{\prime}$ such that $S^{\prime}$ is a $\{1, \ldots, c\}$-colorful independent set, then we can simply take $S^{\prime \prime}=S^{\prime}$. For the reverse direction, suppose $G^{\prime}$ has an $s-t$ separator $S^{\prime \prime}$ of size at most $c$ such that $S^{\prime \prime}$ is an independent set that is $C^{\prime}$-colorful for some $C^{\prime} \subseteq\{1, \ldots, c\}$. Recall that all the vertices with colors $1, \ldots, c$ form an independent set in $G^{\prime}$, and that $G^{\prime}$ contains at least one $\{1, \ldots, c\}$-colorful independent set $S^{*}$. Hence we can extend $S^{\prime \prime}$ into a solution $S^{\prime}$ by adding to $S^{\prime \prime}$ exactly those vertices of $S^{*}$ that have a color from $\{1, \ldots, c\} \backslash C^{\prime}$. This finishes the proof of Claim 2 .

Note that the $s-t$ separator $S^{\prime \prime}$ in Claim 2 has the property "being an independent set of size at most $c$ all whose vertices have a different color from $\{1, \ldots, c\}$ ". This is a hereditary property. Consequently, we can decide if such a separator $S^{\prime \prime}$ exists in $g(|V(H)|) \cdot(n+m)$ time by Theorem 1, as Marx et al. [25] point out that Theorem 1 remains true for graphs having a fixed finite number of colors (since $c+1 \leq|V(H)|+1$, the number of colors in the graphs 
we consider does not depend on $n$ ). Claims 1 and 2 imply that $S^{\prime \prime}$ exists if and only if $\psi$ is $H$-good, which completes the proof.

We now use a variant of the color coding technique to show that PATTERN SEPARATOR is FPT parameterized jointly by the number of vertices in $H$ and the maximum $\operatorname{degree}$ of $G$.

Theorem 11. Pattern Separator can be solved in $f(|V(H)|, \Delta(G)) \cdot m \log n$ time.

Proof. Let $(G, H, s, t)$ be an instance of Pattern Separator. We define $k=|V(H)|$ and $\Delta=\Delta(G)$. Suppose $(G, H, s, t)$ is a yes-instance, and let $S$ be an $s-t$ separator of $G$ such that $G[S]$ is isomorphic to $H$. Let $H_{1}, \ldots, H_{c}$ be the connected components of $H$, and let $S_{i} \subseteq S$ be such that $G\left[S_{i}\right]$ is isomorphic to $H_{i}$ for $1 \leq i \leq c$. Since $|S|=|V(H)|=k$, and every vertex in $S$ has at most $\Delta$ neighbors, $\left|N_{G}[S]\right| \leq(\Delta+1) k$. As a result of Theorem 3 , we can construct a $(\Delta+1) k$-perfect family $\Psi$ of hash functions from $\{1, \ldots, n\}$ to $\{1, \ldots,(\Delta+1) k\}$, consisting of $2^{O((\Delta+1) k)} \cdot \log n$ hash functions. We will interpret each hash function in $\Psi$ as a $(\Delta+1) k$-coloring of $G$. We construct a larger family of colorings $\Psi^{\prime}$ by adding to $\Psi$, for every coloring $\psi \in \Psi$, all colorings that can be obtained from $\psi$ by permuting the colors. Since the total number of colors in any coloring in $\Psi$ is at most $(\Delta+1) k$, the new family $\Psi^{\prime}$ has at most $((\Delta+1) k) ! \cdot 2^{O((\Delta+1) k)} \cdot \log n$ colorings.

In order to use Lemma 6 , we need to transform the $(\Delta+1) k$-colorings in $\Psi^{\prime}$ into $(c+1)$ colorings. For every coloring $\psi^{\prime} \in \Psi^{\prime}$, we define a $(c+1)$-coloring $\psi^{\prime \prime}$ by assigning color 1 to every vertex $v \in V(G)$ for which $1 \leq \psi^{\prime}(v) \leq\left|V\left(H_{1}\right)\right|$, color $i$ to every $v$ for which $1+$ $\sum_{j=1}^{i-1}\left|V\left(H_{j}\right)\right| \leq \psi^{\prime}(v) \leq \sum_{j=1}^{i}\left|V\left(H_{j}\right)\right|$ for every $2 \leq i \leq c$, and color $c+1$ to every $v$ for which $|V(H)|+1 \leq \psi^{\prime}(v) \leq(\Delta+1) k$. Let $\Psi^{\prime \prime}$ be the family of all colorings $\psi^{\prime \prime}$ thus constructed. We claim that $\Psi^{\prime \prime}$ contains an $H$-good coloring. By the definition $\Psi$, there exists a $\xi \in \Psi$ that colors every vertex in $N_{G}[S]$ with a different color. Since $\Psi^{\prime}$ contains all colorings that can be obtained from $\xi$ by permuting the colors, there exists a coloring $\xi^{\prime} \in \Psi^{\prime}$ that satisfies the following properties: $1 \leq \xi^{\prime}(v) \leq\left|S_{1}\right|$ for every $v \in S_{1}, 1+\sum_{j=1}^{i-1}\left|S_{j}\right| \leq \xi^{\prime}(v) \leq \sum_{j=1}^{i}\left|S_{j}\right|$ for every $v \in S_{i}$, and $|S|+1 \leq \xi^{\prime}(v) \leq(\Delta+1) k$ for every $v \in N_{G}(S)$. Let $\xi^{\prime \prime} \in \Psi^{\prime \prime}$ be the $(c+1)$-coloring obtained from $\xi^{\prime}$ in the way described above. It follows from Definition 1 that $\xi^{\prime \prime}$ is $H$-good.

From the above it is clear that, given an instance $(G, H, s, t)$ of PATtern Separator where $G$ has maximum degree at most $\Delta$, we can construct a family $\Psi^{\prime \prime}$ of $(c+1)$-colorings of $G$ such that $\Psi^{\prime \prime}$ contains an $H$-good coloring if and only if $(G, H, s, t)$ is a yes-instance. The size of $\Psi^{\prime \prime}$ is bounded by $((\Delta+1) k) ! \cdot 2^{O((\Delta+1) k)} \cdot \log n$, and $\Psi^{\prime \prime}$ can be constructed in $((\Delta+1) k) ! \cdot 2^{O((\Delta+1) k)} \cdot n \log n$ time by Theorem 3 . Finally, we can check for each coloring in $\Psi^{\prime \prime}$ whether or not it is $H$-good in $g(k) \cdot(n+m)$ time by Lemma 6 for some function $g$ that does not depend on $n$. This yields an overall running time of $f(k, \Delta) \cdot m \log n$ for some function $f$ that only depends on $k$ and $\Delta$.

We now use Theorem 11 to show that the $\mathcal{G}$-SEPARATOR problem is FPT parameterized jointly by the size of the separator and the maximum degree of the input graph $G$.

Theorem 12. For any decidable class $\mathcal{G}$, the $\mathcal{G}$-SEPARATOR problem can be solved in $h_{\mathcal{G}}(k, \Delta(G))$. $m \log n$ time.

Proof. Let $(G, s, t, k)$ be an instance of $\mathcal{G}$-SEPARATOR. We generate all graphs $H$ on at most $k$ vertices that belong to $\mathcal{G}$. Note that there are at most $2^{k^{2}}$ such graphs $H$, and since $\mathcal{G}$ is decidable, we can generate all of them in $f_{\mathcal{G}}(k)$ time for some function $f_{\mathcal{G}}$. For each of the generated graphs $H$, we solve PATTERN SEPARATOR with the graphs $G$ and $H$ as input. This can be done in $f(k, \Delta(G)) \cdot m \log n$ time for each graph $H$ due to Theorem 11. It is clear that 
$(G, s, t, k)$ is a yes-instance of $\mathcal{G}$-SEPARATOR if and only if one of the constructed graphs $H$ yields a yes-instance of PATtern SePARATOR. The overall running time of this algorithm is $h_{\mathcal{G}}(k, \Delta(G)) \cdot m \log n$ for some function $h_{\mathcal{G}}$ that only depends on $k$ and $\Delta(G)$.

\section{No Polynomial Kernel for Connected Separator}

As mentioned in Section 1, Marx et al. [25] showed that the Connected Separator problem is FPT. In Section 2, we proved that the same holds for the problems 2-Connected SEPARATOR and 2-ConneCted Steiner. In this section, we first show that none of these problems admits a polynomial kernel, unless NP $\subseteq$ coNP/poly. We then prove that, under the same assumption, Connected SeParator does not even admit a polynomial kernel when restricted to graphs of maximum degree at most 3.

Let us first introduce some additional terminology and describe two recently established techniques for proving the non-existence of polynomial kernels. For a parameterized problem $Q \subseteq \Sigma^{*} \times \mathbb{N}$, its unparameterized version $\tilde{Q}$ is the set $\left\{x \mathbf{1}^{k} \mid(x, k) \in Q\right\}$, where $\mathbf{1} \notin \Sigma$.

Definition 2. An or-composition algorithm for a parameterized problem $Q \subseteq \Sigma^{*} \times \mathbb{N}$ is an algorithm that receives as input a sequence $\left(\left(x_{1}, k\right), \ldots,\left(x_{r}, k\right)\right)$, with $\left(x_{i}, k\right) \in \Sigma^{*} \times \mathbb{N}^{+}$for each $1 \leq i \leq r$, and outputs a pair $\left(x^{\prime}, k^{\prime}\right)$, such that

- the algorithm uses time polynomial in $\sum_{i=1}^{r}\left|x_{i}\right|+k$;

- $k^{\prime}$ is bounded by a polynomial in $k$; and

- $\left(x^{\prime}, k^{\prime}\right) \in Q$ if and only if there exists an $i \in\{1, \ldots, r\}$ with $\left(x_{i}, k\right) \in Q$.

A parameterized problem $Q$ is said to be or-compositional if there exists an or-composition algorithm for $Q$.

Combining results by Bodlaender et al. [2] and Fortnow and Santhanam [16] yields the following theorem, which provides the first framework for proving the non-existence of polynomial kernels.

Theorem $13([\mathbf{2}],[\mathbf{1 6}])$. Let $Q$ be a parameterized problem and let $\tilde{Q}$ be the corresponding unparameterized decision problem. If $\tilde{Q}$ is $N P$-complete and $Q$ is or-compositional, then $Q$ has no polynomial kernel, unless $N P \subseteq$ coNP/poly.

A second framework for proving the non-existence of polynomial kernels was introduced by Bodlaender, Thomassé and Yeo [4]. A polynomial parameter transformation from a parameterized problem $P$ to a parameterized problem $Q$ is a polynomial-time function that transforms each instance $(x, k)$ of $P$ into an instance $\left(x^{\prime}, k^{\prime}\right)$ of $Q$, such that $(x, k)$ is a yes-instance if and only if $\left(x^{\prime}, k^{\prime}\right)$ is a yes-instance, and $k^{\prime} \leq p(k)$ for some polynomial $p$.

Theorem 14 ([6]). Let $P$ and $Q$ be parameterized problems, and let $\tilde{P}$ and $\tilde{Q}$ be the unparameterized versions of $P$ and $Q$, respectively. Suppose that $\tilde{P}$ is $N P$-complete and $\tilde{Q}$ is in $N P$. If there is a polynomial parameter transformation from $P$ to $Q$ and $Q$ has a polynomial kernel, then $P$ also has a polynomial kernel.

In Section 2, we gave two simple polynomial-time reductions to show that each of the problems Connected Separator, 2-Connected Separator and 2-Connected Steiner is NP-complete. By observing that these two reductions are in fact polynomial parameter transformations, Theorem 14 readily implies the following result.

Proposition 1. None of the problems Connected Separator, 2-Connected Separator and 2-ConneCted SteIner admits a polynomial kernel, unless $N P \subseteq$ coNP/poly. 
In the remainder of this section, we show that ConNected SePARATor does not admit a polynomial kernel, even when restricted to graphs with maximum degree at most 3, unless NP $\subseteq$ coNP/poly. In order to be able to invoke Theorem 13, we first show that the problem remains NP-complete under this restriction (Theorem 15 below). In fact, we prove that ConNECTED SEPARATOR is NP-complete if we assume the input graph not only to have maximum degree at most 3 , but also to possess some additional structure. This structure is then exploited in the proof of Theorem 16, where we show that Connected Separator is or-compositional. We first introduce some additional terminology and prove a useful lemma.

Let $v$ be a vertex of a graph $G$ with degree at least 4 , and let $\left\{w_{1}, \ldots, w_{p}\right\}$ denote the neighbors of $v$ in $G$. When we say that we blow up vertex $v$, we first delete $v$ from $G$, and then add a cycle $C^{v}$ with vertex set $\left\{c_{1}, \ldots, c_{p}\right\}$ as well as the edge $c_{i} w_{i}$ for every $1 \leq i \leq p$. For any graph $G$ and integer $\ell$, we define the graph $G_{\ell}$ as the graph that can be obtained from $G$ by first subdividing every edge of $G$ exactly $\ell$ times, and then blowing up every vertex that has degree at least 4 . For every edge $u v \in E(G)$, we refer to the set $X^{u v}$ of vertices that were inserted to subdivide the edge $u v$ as white vertices in $G_{\ell}$. For every vertex $v \in V(G)$ of degree at least 4 in $G$, we refer to the vertices of $C^{v}$ as black vertices in $G_{\ell}$. Every vertex in $G$ that has degree at most 3 is not blown up, and therefore appears as a vertex in $G_{\ell}$. For convenience, we define $C^{w}=\{w\}$ for every $w \in V(G)$ of degree at most 3, and we say that $w$ is a black vertex in $G_{\ell}$.

Lemma 7. Let $G$ be a graph with maximum degree $\Delta$, and let $s$ and $t$ be two vertices of degree 2 in $G$. Let $\ell=\Delta k+1$. Then $G$ has a connected $s-t$ separator of size at most $k$ if and only if $G_{\ell}$ has a connected $s-t$ separator of size at most $(k-1) \ell+\Delta k$.

Proof. Since $s$ and $t$ have degree 2 in $G$, they are not blown up when creating $G_{\ell}$ from $G$. Hence $s$ and $t$ appear as black vertices of degree 2 in $G_{\ell}$.

Suppose $G$ has a connected $s-t$ separator $S$ of size at most $k$. Let $T$ be a spanning tree of the graph $G[S]$. Let $S^{\prime} \subseteq V\left(G_{\ell}\right)$ be the set containing all the vertices of $C^{u}$ for every vertex $u \in S$, as well as all the vertices of $X^{u v}$ for every edge $u v \in E(T)$. Since $T$ contains at most $k$ vertices and therefore at most $k-1$ edges, $S^{\prime}$ contains at most $\Delta k$ black vertices and at most $(k-1) \ell$ white vertices, so $\left|S^{\prime}\right| \leq(k-1) \ell+\Delta k$. It follows from the construction of $G_{\ell}$ that $S^{\prime}$ is a connected $s-t$ separator in $G_{\ell}$.

For the reverse direction, suppose $G_{\ell}$ has a connected $s-t$ separator $S^{\prime}$ of size at most $(k-1) \ell+\Delta k$. We assume that $S^{\prime}$ is minimal, i.e., no proper subset $S^{\prime \prime} \subset S^{\prime}$ is a connected $s-t$ separator in $G^{\prime}$. We show that, for every edge $u v$ in $G, S^{\prime}$ either contains none of the vertices of $X^{u v}$, or contains all the vertices of $X^{u v}$, as well as the two black vertices (one from $C^{u}$ and one from $C^{v}$ ) that are adjacent to $X^{u v}$. For contradiction, suppose that this is not the case for some edge $u v \in E(G)$. Then $S^{\prime}$ contains a white vertex $x$ that has degree 1 in $G_{\ell}\left[S^{\prime}\right]$, which implies that $S^{\prime} \backslash\{x\}$ is also a connected $s-t$ separator, contradicting the minimality of $S^{\prime}$. Let $X$ be the set of edges $u v$ in $G$ for which all vertices of $X^{u v}$ are contained in $S^{\prime}$, i.e., $X=\left\{u v \in E(G) \mid X^{u v} \subseteq S^{\prime}\right\}$. Suppose that $|X| \geq k$. Then $S^{\prime}$ contains at least $k \ell$ white vertices, i.e., $\left|S^{\prime}\right| \geq k \ell=(k-1) \ell+\ell$. Since $\ell=\Delta k+1$, this contradicts the assumption that $\left|S^{\prime}\right| \leq(k-1) \ell+\Delta k$. Hence $X$ contains at most $k-1$ edges. Let $S \subseteq V(G)$ be the set of endpoints of the edges in $X$. Since $S^{\prime}$ is a connected set in $G^{\prime}$, the graph $G[S]$ is connected. Moreover, since every connected graph on at most $k-1$ edges contains at most $k$ vertices, $S$ contains at most $k$ vertices. From the construction of $G_{\ell}$ and the fact that $S^{\prime}$ is an $s-t$ separator in $G_{\ell}$, it follows that $S$ is an $s^{-} t$ separator in $G$.

Theorem 15. The Connected SePARATOR problem is NP-complete on graphs of maximum degree at most 3 , in which the vertices $s$ and $t$ have degree 2 . 


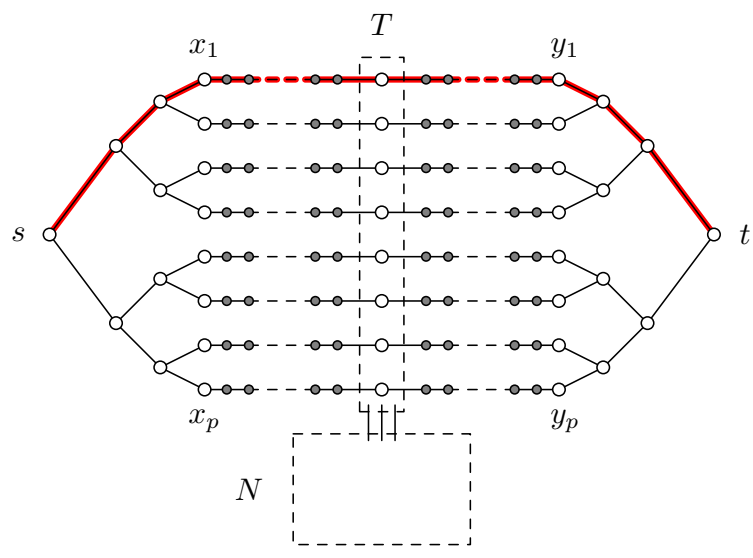

Fig. 3. The graph $G^{\prime}$ constructed from an instance $(G, T, k)$ of Steiner Tree, where $N=V(G) \backslash T$. The graph $G^{\prime}[T \cup N]$ is isomorphic to $G$. The vertices of $X^{\prime}$ and $Y^{\prime}$ are colored grey. The unique path $Q$ from $s$ to $t$ that contains no vertices from $(T \cup N) \backslash\left\{t_{1}\right\}$ is indicated using bold (red) edges.

Proof. We first show that Connected SEPARATOR is NP-complete when $s$ and $t$ have degree 2, before using Lemma 7 to prove that the problem remains NP-complete with the additional restriction that the maximum degree of the input graph is at most 3 . Let $(G, T, k)$ be an instance of Steiner Tree, where $T=\left\{t_{1}, \ldots, t_{p}\right\}$. We define $N=V(G) \backslash T$ to be the set of non-terminals in $G$. We assume that $T$ is an independent set in $G$, and point out that the problem remains NP-complete under this assumption [21]. We also assume that $p=2^{q}$ for some integer $q$; if this is not the case, then we simply add an independent set $X$ of new vertices to $G$ such that $|T \cup X|=2^{q}$ for some integer $q$, making each of them a false twin of $t_{1}$ by adding edges between every vertex in $X$ and every vertex in $N_{G}\left(t_{1}\right)$.

We transform $(G, T, k)$ into an instance $\left(G^{\prime}, s, t, k\right)$ of Connected Separator as follows; see Figure 3 for a helpful illustration. To construct $G^{\prime}$, we start with a copy of $G$. For convenience, we use $T$ and $N$ to refer to the vertices in $G^{\prime}$ corresponding to the terminals and non-terminals of $G$, respectively. We add independent sets of vertices $X=\left\{x_{1}, \ldots, x_{p}\right\}$ and $Y=\left\{y_{1}, \ldots, y_{p}\right\}$, as well as the edges $t_{i} x_{i}$ and $t_{i} y_{i}$ for every $1 \leq i \leq p$. We then subdivide every edge of the form $t_{i} x_{i}$ exactly $k$ times, and we use $X^{\prime}$ to denote the set of vertices of degree 2 that are inserted to subdivide these edges. We do the same for every edge of the form $t_{i} y_{i}$, and define the set $Y^{\prime}$ accordingly. We add a vertex $s$, and connect $s$ to the vertices in $X$ using a binary tree of depth $q$, whose root is $s$ and whose leaves are exactly the vertices of $X$. Let $V_{X}$ be the set of vertices contained in the binary tree, along with the vertices of $X^{\prime}$. We now add a vertex $t$, and connect $t$ to the vertices of $Y$ in the same way. The set $V_{Y}$ is defined analogously to $V_{X}$. This finishes the construction of $G^{\prime}$. Note that the sets $V_{X}, T \cup N$ and $V_{Y}$ form a partition of $V\left(G^{\prime}\right)$. We show that $\left(G^{\prime}, s, t, k\right)$ is a yes-instance of Connected SEParator if and only if $(G, T, k)$ is a yes-instance of Steiner Tree.

Suppose there exists a subset $N^{\prime} \subseteq N$ such that $\left|T \cup N^{\prime}\right| \leq k$ and the graph $G\left[T \cup N^{\prime}\right]$ is connected. Every path from $s$ to $t$ contains at least one vertex of $T$, so $T \cup N^{\prime}$ forms a connected $s-t$ separator in $G^{\prime}$. Since $\left|T \cup N^{\prime}\right| \leq k,\left(G^{\prime}, s, t, k\right)$ is a yes-instance of ConneCted SEPARATOR.

For the reverse direction, suppose there exists a connected $s-t$ separator of size at most $k$ in $G^{\prime}$. Let $S$ be such a separator, and assume that $S$ is minimal, i.e., no proper subset $S^{\prime} \subset S$ is a connected $s-t$ separator in $G^{\prime}$. Due to the minimality of $S$, we know that $S$ does not contain a vertex $x$ that has degree 1 in $G^{\prime}[S]$ and degree 2 in $G^{\prime}$, as then $S \backslash\{x\}$ would also be a 
connected $s-t$ separator. All vertices in $X^{\prime}$ have degree 2 in $G^{\prime}$, and are thus only contained in $S$ if both neighbors are contained in $S$. Since $X^{\prime}$ consists of paths on $k$ vertices of degree 2 in $G^{\prime}, S \cap X^{\prime} \neq \emptyset$ would imply that $S$ contains at least $k+2$ vertices. This would contradict the assumption $|S| \leq k$, so we conclude that $S \cap X^{\prime}=\emptyset$. By symmetric arguments, we also have that $S \cap Y^{\prime}=\emptyset$.

Let $Q$ (respectively $R$ ) be the unique path in $G^{\prime}$ from $s$ to $t$ that contains no vertices from $(T \cup N) \backslash\left\{t_{1}\right\}$ (respectively $(T \cup N) \backslash\left\{t_{p}\right\}$ ); the path $Q$ is indicated using bold (red) edges Figure 3. Any $s-t$ separator contains at least one vertex of every path between $s$ and $t$. In particular, $S$ contains at least one vertex from $Q$ and at least one vertex from $R$. Let $q \in S \cap V(Q)$ and $r \in S \cap V(R)$. Suppose, for contradiction, that $q \in V_{X} \backslash X^{\prime}$. Since $S$ is a connected $s-t$ separator, the graph $G[S]$ contain a path from $q$ to $r$. By the construction of $G^{\prime}$, such a path must contain at least $k$ vertices of $X^{\prime}$, as well as $q$ and $r$. This contradicts the assumption that $|S| \leq k$. This contradiction implies that $S \cap V_{X} \backslash X^{\prime}=\emptyset$. Then we also have $S \cap X^{\prime}=\emptyset$ by the minimality of $S$, so $q \in T$. Using symmetry, we also find that $r \in T$. Considering other pairs $Q^{\prime}, R^{\prime}$ of internally vertex-disjoint paths from $s$ to $t$, we can use similar arguments to show that $S \cap\left(V_{X} \cup V_{Y}\right)=\emptyset$, and that $S$ contains every vertex of $T$. Since $|S| \leq k$, at most $k-|T|$ vertices of $S$ belong to $N$. Let $N^{\prime}=S \cap N$, i.e., $S=T \cup N^{\prime}$. Since $S$ is a connected $s-t$ separator, $G\left[T \cup N^{\prime}\right]$ is connected. Hence $(G, T, k)$ is a yes-instance of STEINER TREE.

We have showed that ConneCTED SEPARATOR is NP-complete when $s$ and $t$ have degree 2 . We now reduce this problem to the CONNECTED SEPARATOR problem on graphs with maximum degree at most 3 as follows. Let $\left(G^{\prime}, s, t, k\right)$ be an instance of ConneCTED SEPARATOR, where $s$ and $t$ have degree 2 in $G^{\prime}$. Let $\Delta$ be the maximum degree in $G^{\prime}$, and let $\ell=\Delta k+1$. We create a new instance $\left(G^{\prime \prime}, s, t, k^{\prime}\right)$ of Connected SePARAtor by defining $G^{\prime \prime}=G_{\ell}$ and $k^{\prime}=(k-1) \ell+\Delta k$. Since we do not blow up vertices of degree less than 4 when transforming $G^{\prime}$ into $G^{\prime \prime}, s$ and $t$ exist and have degree 2 in $G^{\prime \prime}$. By Lemma $7, G^{\prime \prime}$ has a connected $s-t$ separator of size at most $k^{\prime}$ if and only if $G^{\prime}$ has a connected $s-t$ separator of size at most $k$. The observation that $G^{\prime \prime}$ has degree at most 3 completes the proof.

Theorem 16. The CONNECTEd SEPARATOR problem, restricted to graphs with maximum degree at most 3 , is or-compositional.

Proof. As we showed in Theorem 15, Connected SeParator is NP-complete on graphs of maximum degree at most 3 , in which both input vertices $s$ and $t$ have degree 2 . In fact, we may assume $s$ and $t$ to be adjacent to the vertices of $T$ by means of binary trees, since no vertex of $V_{X} \cup V_{Y}$ was blown up in the construction of $G_{\ell}$ in the proof of Theorem 15. We may also assume that the graph $G-\{s, t\}$ is connected, since otherwise we have a trivial no-instance, which may be safely discarded from the sequence of instances in the input of the composition algorithm. We say that an instance $(G, s, t, k)$ of Connected Separator is pretty when it satisfies all the structural properties described above.

Let $\left(\left(G_{1}, s_{1}, t_{1}, k\right), \ldots,\left(G_{r}, s_{r}, t_{r}, k\right)\right)$ be a sequence of pretty inputs of the ConNECTED Separator problem. For every $1 \leq i \leq r$, let $s_{i}^{\prime}, s_{i}^{\prime \prime}$ and $t_{i}^{\prime}, t_{i}^{\prime \prime}$ be the neighbors of $s_{i}$ and $t_{i}$, respectively. We create an instance $(G, s, t, k)$ of Connected Separator as follows. To construct $G$ from the graphs $G_{1}, \ldots, G_{r}$, we delete, for every $1 \leq i \leq r-1$, the vertices $t_{i}$ and $s_{i+1}$, add edges $t_{i}^{\prime} s_{i+1}^{\prime}$ and $t_{i}^{\prime \prime} s_{i+1}^{\prime \prime}$, and subdivide both these edges $k+1$ times. Let $Z_{i}^{\prime}$ and $Z_{i}^{\prime \prime}$ denote the set of vertices that are used to subdivide the edges $t_{i}^{\prime} s_{i+1}^{\prime}$ and $t_{i}^{\prime \prime} s_{i+1}^{\prime \prime}$, respectively. We write $V_{i}$ to denote the set of vertices in $G$ corresponding to the graph $G_{i}$; in particular, $V_{i}=V\left(G_{i}\right) \backslash\left\{s_{i}, t_{i}\right\}$ for every $2 \leq i \leq r-1$. Finally, let $s=s_{1}$ and $t=t_{r}$. See Figure 4 for an illustration of the graph $G$.

We now show that $(G, s, t, k)$ is a yes-instance of Connected Separator if and only if there exists an $i \in\{1, \ldots, r\}$ such that $\left(G_{i}, s_{i}, t_{i}, k\right)$ is a yes-instance. If $G_{i}$ has a connected 


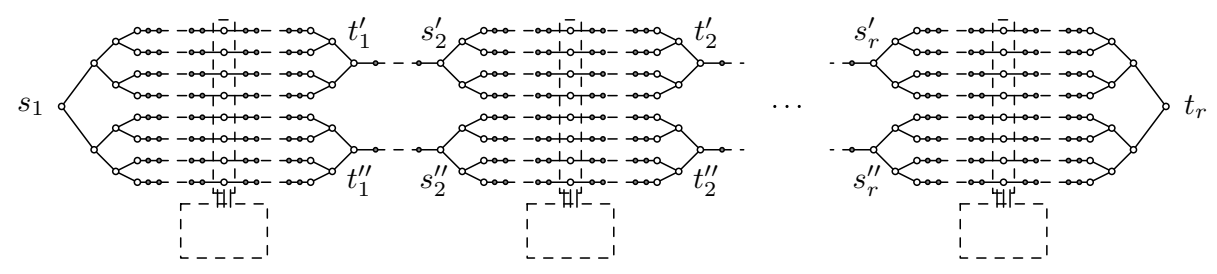

Fig. 4. The graph $G$ constructed in the or-composition in the proof of Theorem 16 .

$s_{i}-t_{i}$ separator $S$ of size at most $k$ for some $i$, then it is clear from the construction of $G$ that $S$ is also an $s_{1}-t_{r}$ separator. Hence we only need to prove that if $(G, s, t, k)$ is a yes-instance of Connected Separator, then $\left(G_{i}, s_{i}, t_{i}, k\right)$ is a yes-instance for some integer $i$.

Suppose $G$ has a connected $s-t$ separator $S$ of size at most $k$. Assume that $S$ is minimal, i.e., no proper subset $S^{\prime} \subset S$ is a connected $s-t$ separator in $G$. As mentioned before, any vertex of degree 2 in $G$ is, due to the minimality of $S$, only contained in $S$ if both neighbors are contained in $S$. Hence, since $\left|Z_{i}^{\prime}\right|=\left|Z_{i}^{\prime \prime}\right|=k+1$ and $|S| \leq k$, we can conclude that $S \cap\left(Z_{i}^{\prime} \cup Z_{i}^{\prime \prime}\right)=\emptyset$ for $1 \leq i \leq r-1$. Suppose that $S$ contains a vertex $v_{i} \in V_{i}$ and a vertex $v_{j} \in V_{j}$ such that $i<j$. Since $S$ is connected, $S$ must contain all the vertices of $Z_{i+1}^{\prime}$ or $Z_{i+1}^{\prime \prime}$. Since $\left|Z_{i+1}^{\prime}\right|=\left|Z_{i+1}^{\prime \prime}\right|=k+1$, this contradicts the assumption that $S$ has size at most $k$. Hence $S \subseteq V_{i}$ for exactly one integer $i$ with $1 \leq i \leq r-1$. It is clear that $S$ is connected in $G_{i}$ and that $S \cap\left\{s_{i}, t_{i}\right\}=\emptyset$. We claim that $S$ is an $s_{i}-t_{i}$ separator in $G_{i}$. For contradiction, suppose there exists an $s_{i}-t_{i}$ path $P$ in $G_{i}-S$. Let $u$ and $v$ denote the neighbors of $s_{i}$ and $t_{i}$ in $P$, respectively. The subpath of $P$ from $u$ to $v$, together with a path from $s_{1}$ to $u$ and a path from $v$ to $t_{r}$, forms a path from $s_{1}$ to $t_{r}$ in $G-S$. This contradicts the assumption that $S$ is an $s-t$ separator in $G$.

The instance $(G, s, t, k)$ can clearly be constructed from the given sequence of instances in time polynomial in $\sum_{i=1}^{r}\left|V\left(G_{i}\right)\right|+k$, and the parameter in $(G, s, t, k)$ is the same as the parameter in each of the given instances $\left(G_{i}, s_{i}, t_{i}, k\right)$. Hence Connected Separator is orcompositional.

Combining results of Bodlaender et al. [2] and Fortnow and Santhanam [16] on the nonexistence of polynomial kernels, together with Theorems 15 and 16, yields the following result.

Theorem 17. The CONNECTED SePARATor problem, restricted to graphs of maximum degree at most 3, has no polynomial kernel, unless $N P \subseteq$ coNP/poly.

\section{Conclusions}

Motivated by recent results due to Marx et al. [24, 25], we studied the parameterized complexity of the $\mathcal{G}$-SEPARATOR problem for certain non-hereditary graph classes $\mathcal{G}$. On the negative side, we showed that the problem is $W$ [1]-hard when $\mathcal{G}$ is the class of $c$-connected graphs for any $c \geq 3$, the class of $r$-regular graphs for any $r \geq 1$ or the class of graphs of diameter at most $d$ for any $d \geq 2$. On the positive side, we showed that the problem is FPT when $\mathcal{G}$ is the class of 2-connected graphs. Marx et al. [25] showed that the same holds when $\mathcal{G}$ is the class of connected graphs. Are there other interesting non-hereditary graph classes $\mathcal{G}$ for which the $\mathcal{G}$-SEPARATOR problem is FPT?

Acknowledgements. We would like to thank Pål Grønås Drange for fruitful discussions. 


\section{References}

1. Alon, N, Yuster, R, Zwick, U.: Color-coding. J. ACM 42, 844-856 (1995)

2. Bodlaender, H.L., Downey, R.G., Fellows, M.R., Hermelin, D.: On problems without polynomial kernels. J. Comput. Syst. Sci. 75, 423-434 (2009)

3. Bodlaender, H.L., Fomin, F.V., Lokshtanov, D., Penninkx, E., Saurabh, S., Thilikos, D.M.: (Meta) Kernelization. In: FOCS 2009, pp. 629-638. IEEE Computer Society (2009)

4. Bodlaender, H.L., Thomassé, S., Yeo, A.: Kernel bounds for disjoint cycles and disjoint paths. Theor. Comput. Sci. 412(35), 4570-4578 (2011)

5. Bousquet, N., Daligault, J., Thomassé, S.: Multicut is FPT. In: Fortnow, L., Vadhan, S.P. (eds.) STOC 2011, pp. 459-468. ACM (2011)

6. Bodlaender, H.L., Thomassé, S., Yeo, A.: Kernel bounds for disjoint cycles and disjoint paths. Theor. Comput. Sci. 412(35), 4570-4578 (2011)

7. Chen, J., Liu, Y., Lu, S.: An improved parameterized algorithm for the minimum node multiway cut problem. Algorithmica 55(1):1-13 (2009)

8. Courcelle, B.: Graph rewriting: an algebraic and logic approach. In: Van Leeuwen (ed.) Handbook of Theoretical Computer Science, Volume B: Formal Models and Semantics, pp. 193-242. Elsevier and MIT Press, Amsterdam (1990)

9. Demaine, E., Hajiaghayi, M., Marx, D.: Minimizing movement: fixed-parameter tractability. In: Fiat, A., Sanders, P. (eds.) ESA 2009. LNCS, vol. 5757, pp. 718-729. Springer (2009)

10. Diestel, R.: Graph Theory. Electronic Edition (2005)

11. Downey, R., Fellows, M.R.: Parameterized Complexity. Monographs in Computer Science, Springer, New York (1999)

12. Dreyfus, S., Wagner, R.: The Steiner problem in graphs. Networks 1:195-207 (1971)

13. Feige, U., Mahdian, M.: Finding small balanced separators. In: Kleinberg, J.M. (ed.) STOC 2006, pp. 375-384. ACM (2006)

14. Fellows, M.R., Hermelin, D., Rosamond, F.A., Vialette, S.: On the parameterized complexity of multiple-interval graph problems. Theoretical Computer Science 410, 53-61 (2009)

15. Fomin, F.V., Lokshtanov, D., Saurabh, S., Thilikos, D.M.: Bidimensionality and kernels. In: Charikar, M. (ed.) SODA 2010, pp. 503-510. SIAM (2010)

16. Fortnow, L., Santhanam, R.: Infeasibility of instance compression and succinct PCPs for NP. J. Comput. Syst. Sci. 77, 91-106 (2011)

17. Gottlob, G., Lee, S.T.: A logical approach to multicut problems. Inform. Process. Lett. 103(4), 136-141 (2007)

18. Guillemot, S.: FPT algorithms for path-transversal and cycle-transversal problems. Discrete Optimization 8(1), 61-71 (2011)

19. Guo, J., Hüffner, F., Kenar, E., Niedermeier, R., Uhlmann, J.: Complexity and exact algorithms for vertex multicut in interval and bounded treewidth graphs. Eur. J. Oper. Res. 186(2), 542-553 (2008)

20. Heggernes, P., van 't Hof, P., Marx, D., Misra, N., Villanger, Y.: On the parameterized complexity of finding separators with non-hereditary properties. In: Golumbic, M.C., Stern, M. (eds.) WG 2012. LNCS, Springer (to appear)

21. Hwang, F.K., Richards, D.S., Winter, P.: Steiner tree problems. Annals of Discrete Mathematics 53, North-Holland (1992)

22. R.M. Karp. Reducibility among combinatorial problems. In: Complexity of Computer Computations, pp. 85-103. Plenum Press, New York (1972)

23. Marx, D.: Parameterized graph separation problems. Theor. Comput. Sci. 351(3), 394-406 (2006)

24. Marx, D., O'Sullivan, B., Razgon, I.: Treewidth reduction for constrained separation and bipartization problems. In: Marion, J.-Y., Schwentick, T. (eds.) STACS 2010, pp. 561-572 (2010)

25. Marx, D., O'Sullivan, B., Razgon, I.: Finding small separators in linear time via treewidth reduction. CoRR, arXiv:1110.4765 (2011). To appear in ACM Transactions on Algorithms.

26. Marx, D., Razgon, I.: Constant ratio fixed-parameter approximation of the edge multicut problem. Inform. Process. Lett. 109(20), 1161-1166 (2009)

27. Marx, D., Razgon, I.: Fixed-parameter tractability of multicut parameterized by the size of the cutset. In: Fortnow, L., Vadhan, S.P. (eds.) STOC 2011, pp. 469-478. ACM (2011)

28. Tarjan, R.E.: Decomposition by clique separators. Discrete Math. 55, 221-232 (1985) 